\title{
Resolution of reservoir scale electrical anisotropy from marine CSEM data
}

\section{Vanessa Brown ${ }^{1,2}$, Mike Hoversten ${ }^{3}$, Kerry Key ${ }^{2}$ and Jinsong Chen ${ }^{4}$}

\author{
${ }^{1}$ Institut de Physique du Globe de Paris, 1 rue Jussieu, Paris, FRANCE \\ ${ }^{2}$ Scripps Institution of Oceanography, University of California San Diego \\ 9500 Gilman Drive, La Jolla, CA, 92093-0225, USA \\ ${ }^{3}$ Chevron Energy Technology Company, San Ramon, CA, USA \\ ${ }^{4}$ LBNL Berkeley, CA, USA \\ Email: brown@,ipgp.fr
}




\begin{abstract}
A combination of $1 \mathrm{D}$ and 3D forward and inverse solutions is used to quantify the sensitivity and resolution of conventional controlled source electromagnetic (CSEM) data collected using a horizontal electric dipole source to transverse electrical anisotropy located in a deep-water exploration reservoir target. Since strongly anisotropic shale layers have a vertical resistivity that can be comparable to many reservoirs, we examine how CSEM can discriminate confounding shale layers through their characteristically lower horizontal resistivity. Forward modeling demonstrates that the sensitivity to reservoir level anisotropy is very low compared to the sensitivity to isotropic reservoirs, especially when the reservoir is deeper than about $2 \mathrm{~km}$ below the seabed. However, for 1D models where the number of inversion parameters can be fixed to be only a few layers, both vertical and horizontal resistivity of the reservoir can be well resolved using a stochastic inversion. We find that the resolution of horizontal resistivity increases as the horizontal resistivity decreases. We show that this effect is explained by the presence of strong horizontal current density in anisotropic layers with low horizontal resistivity. Conversely, when the reservoir has a vertical to horizontal resistivity ratio of about 10 or less, the current density is vertically polarized and hence has little sensitivity to the horizontal resistivity. Resistivity anisotropy estimates from 3D inversion for $3 \mathrm{D}$ targets suggest that resolution of reservoir level anisotropy for 3D targets will require good a priori knowledge of the background sediment conductivity and structural boundaries.
\end{abstract}




\section{INTRODUCTION}

The field of marine controlled source electromagnetics (CSEM) has made major advances in most aspects of data acquisition and interpretation over the last ten years. A significant step in interpretation capability came with the recognition of the importance electrical anisotropy in the measured CSEM responses. The effect of electrical anisotropy has been the subject of many papers: in deep crustal studies (Everett and Constable, 1999), fracture detection and mapping (Le Masne and Vasseur, 1981), mineral exploration (Mansour al-Garni and Everett, 2003), and in borehole logging (Lu and Alumbaugh, 2001). Barber et al. (2004) demonstrate how electrical anisotropy can be determined through borehole logging. Ellis et al. (2010) developed an effective medium model for sediment anisotropy arising through preferred grain shape and alignment.

Initial recognition of the importance of electrical anisotropy for marine CSEM has focused on the relatively modest macro-scale anisotropy where the ratio of vertical $\left(\mathrm{R}_{\mathrm{vert}}\right)$ to horizontal $\left(\mathrm{R}_{\text {horz }}\right)$ resistivity ranges between 1 and 3. The effect of this macro-scale electrical anisotropy in hydrocarbon exploration has been shown to be significant (e.g. Tompkins, 2004, 2005; Hoversten et al. 2006; Lu and Xia 2007). The overburden anisotropy has been shown to produce larger effects than anisotropy at the reservoir level (e.g., Tompkins, 2005, Li and Dai, 2011). Newman et al. (2010) showed that accounting for electrical anisotropy in the background model was essential in fitting offline, or the socalled broadside, CSEM data. More importantly, the ability to fit the broadside data improved the inverted resistivity structure overall. 
The ability to determine both the vertical and horizontal resistivity of reservoir sized zones has several applications. In the case of hydrocarbon saturated sands with homogeneous grain size, where the electrical resistivity is expected to be more isotropic, the ability to accurately image electrical anisotropy could help in distinguishing oil sand from anisotropic shale. Shale is the most common rock where anisotropy (both acoustic and electric) is generated at the micro-scale (although layered shalesand could have macro-scale anisotropy). Hoversten et al. (2006) presented a case where a highly anisotropic shale layer $\left(\mathrm{R}_{\mathrm{ver}} / \mathrm{R}_{\text {horz }} \sim 40\right)$ had been mistaken for a hydrocarbon bearing sand. In comparison, the vertical resistivity of many hydrocarbon-saturated sands can be on the order of $30 \Omega \mathrm{m}$ and in some cases lower, placing them in the same range as anisotropic shale. Accurate determination of horizontal resistivity could help discriminate between a uniform porosity and uniform grain size sands and highly anisotropic shale.

A second application is for the large electrical anisotropy that has also been observed in clean sands with uniform porosity. Anderson et al. (1994) describe anisotropies on the order of 10:1 and greater in such sands where the anisotropy is thought to be caused by variations in grain size and hence permeability (Klein, 1996). Sands with uniform porosity but variable permeability and grain size are electrically isotropic when filled with pore water, only becoming anisotropic with the introduction of hydrocarbons. This leads to the intriguing possibility of making permeability estimates in hydrocarbon filled sands from inferred electrical anisotropy if such an inference were possible. This also means that the transition from regions of isotropic resistivity to regions of anisotropic resistivity would mark the transition from water to oil within reservoir sands, as demonstrated by Klein et al. (1997). A third related application is the use of the horizontal and vertical resistivity as independent data in stochastic rock physics simulations of fluid type, where the ability to accurately determine horizontal resistivity is essential. 
The electromagnetic field originating from a horizontal electric dipole (HED) source can be decomposed into two modes, transverse electric (TE) and transverse magnetic (TM), where transverse refers to the field orthogonal to some symmetry axis. For 1D models, the symmetry is about the vertical axes. The TE mode is characterized by horizontal electric current loops, where coupling between layers is purely inductive; the TM mode is characterized by vertical electric current loops (and horizontal magnetic field loops), where coupling between layers is both galvanic and inductive. When the source and receivers are inline (i.e., the source dipole is pointing along the receiver line) a thin resistive reservoir can be detected by an anomalous electromagnetic field at source receiver offsets typically about 3-6 times the target depth (e.g., Orange et al., 2009). If the electric field is recorded by receivers in the purely broadside (offline) configuration (i.e., the source to receiver line makes a 90 degree angle with the dipole), the anomalous electric field due to the resistive target layer is significantly lower than the inline case (e.g., Constable and Weiss, 2006). Inspection of the field line shape from a HED source shows there are more vertical electric field lines per unit area through the buried resistor directly below and inline with the source compared to offline. The electric fields measured inline will be dominated by the TM mode with a much smaller TE component. The resistive layer acts as an efficient barrier shielding deeper conductors from the TM mode vertical current loops and generates the detectable anomalous response. Conversely, beneath the broadside receivers there are more horizontal field lines through the buried resistor, therefore the TE mode will contribute more to the broadside and offline data. Despite the high resistivity of a reservoir, its thinness precludes significant inductive attenuation; hence the horizontally polarized TE energy is relatively unmodified by its presence. Consequently, it is generally considered that TM mode and galvanic effects are key in generating the anomalous field and hence allowing for the detection of the resistor (e.g., Weidelt, 2007). In contrast to the TE and TM modes produced by a HED source, a vertical electric dipole source 
(VED) will produce only a TM mode and therefore offers lower resolution of target layers (e.g., Key 2009). For higher dimensional features, the TE mode description may no longer be valid in the vicinity of the anomalous structure due to galvanic effects generated along its lateral edges.

If anisotropy is present the electromagnetic fields detected can be further modified. Ramananjaona (2010) demonstrates that the TM mode is sensitive to the vertical resistivity and the anisotropic ratio, while the TE mode is more sensitive to the horizontal resistivity. By examining the reflection coefficients, Ramananjaona (2010) shows that an anisotropic layer of resistivity $\left(\mathrm{R}_{\text {horz }}, \mathrm{R}_{\text {vert }}\right)$ and thickness $\mathrm{H}$, has an equivalent isotropic layer for each mode. For the TE mode, the equivalent isotropic layer is thickness $\mathrm{H}^{\prime}=\mathrm{H}$ and resistivity $\mathrm{R}^{\prime}=\mathrm{R}_{\text {horz }}$, while for the $\mathrm{TM}$ mode the equivalent layer has resistivity $R^{\prime}=R_{\text {vert }}$ and thickness $H^{\prime}=\lambda H$. The $\lambda$ term, which is the anisotropy ratio $\left(\sqrt{ } R_{\text {vert }} / R_{\text {horz }}\right)$, leads to this non-complete equivalence between the isotropic and the anisotropic model, supporting the argument that isotropic modeling can be insufficient for very anisotropic structures.

Overall, HED sources, which generate both TM and TE modes, appear to be more sensitive to vertical resistivity (whether in the overburden, basement or in the reservoir) than horizontal resistivity. Sensitivity to horizontal resistivity, to whatever extent it exists, arises from horizontal current flow from either the inductive component associated with TE mode propagation or from the horizontal part of the TM field. To our knowledge, Abubakar et al. (2010) present the only work to date that has specifically addressed the ability to resolve electrical anisotropy of the reservoir. This work was done using 2D models and only considered data that is inline with either electric or magnetic transmitters. They concluded that both horizontal magnetic dipole sources with horizontal magnetic field receivers and horizontal electric dipole sources with horizontal electric field receivers are required to adequately 
discriminate both horizontal and vertical resistivity. However, their work did not consider the sensitivity of offline data from horizontal electric dipole sources. While the benefits of a magnetic dipole source for determining the horizontal reservoir resistivity are suggested by Abubakar et al. (2010), there is currently no commercial system available with such a source. We therefore have chosen to study the ability, or lack thereof, to determine vertical and horizontal resistivity in reservoirs using only commercially available inline and offline data configurations (i.e., that obtained from horizontal electric dipole sources)

We begin with 1D modeling studies demonstrating the magnitude and location of sensitivity to anisotropy in a deep target example. We also include the same type of study for a 3D slab model example. We then use stochastic 1D inversion simulations and 2D finite element modeling to characterize how well various reservoir anisotropies can be resolved. We finish this work with 3D deterministic inversions of anisotropic versus isotropic deep targets to further characterize how well anisotropy may be resolved in a more realistic data set.

\section{D SENSITIVITY STUDY}

In this section we use layered 1D models to investigate the sensitivity of simulated HED CSEM data to horizontal anisotropy located only in a target reservoir layer. The models, shown in Figure 1 and described in Table 1, contain an anisotropic $100 \mathrm{~m}$ thick target layer at depths of 1,2 and $3 \mathrm{~km}$ below the mud line (BML) in an isotropic host (models 1, 2 and 3 respectively). For comparison, data is also simulated for a 3D reservoir slab model identical to model 2 except the target is a 5 by $5 \mathrm{~km} \mathrm{slab}$ at 2 $\mathrm{km}$ BML. In the target layer, $\mathrm{R}_{\mathrm{vert}}$ is fixed at $40 \Omega \mathrm{m}$ and $\mathrm{R}_{\text {horz }}$ is varied from 1 to $40 \Omega \mathrm{m}$ to allow a 
range of anisotropies, with the end members representing a homogeneous shale and a homogeneous oil filled sand, respectively. The simulated data-acquisition geometry is a single receiver located at the origin. The source is an $\mathrm{x}$-directed horizontal electric dipole (HED) traversing on seventeen east-west sail lines with $\mathrm{x}$ range from -15 to $+15 \mathrm{~km}$. The sail lines are spaced $1 \mathrm{~km}$ apart in the $\mathrm{y}$ direction with the sail line array centered on the receiver location. Source points on each sail line are spaced 250 meters apart. Five frequencies $(0.125,0.25,0.5,1,2 \mathrm{~Hz})$ representative of common field acquisition are simulated. Received electric fields that are parallel to the source dipole $\left(E_{\mathrm{x}}\right)$ are referred to as maxcoupled while electric fields orthogonal to the source dipoles $\left(\mathrm{E}_{\mathrm{y}}\right)$ are referred to as null-coupled.

Figure 2 shows the $\mathrm{E}_{\mathrm{x}}$ and $\mathrm{E}_{\mathrm{y}}$ field amplitude for model 2 with no target layer, with an isotropic target layer $\left(\mathrm{R}_{\text {iso }}=40 \Omega \mathrm{m}\right)$ and with an anisotropic target layer $\left(\mathrm{R}_{\text {vert }}=40 \Omega \mathrm{m}\right.$ and $\left.\mathrm{R}_{\text {horz }}=1 \Omega \mathrm{m}\right)$. Data at $0.5 \mathrm{~Hz}$ is shown because it is the frequency of maximum responses for this model. Figure 2 shows the difference between the target layer and the no target layer case is clear for the $E_{x}$ amplitudes and more difficult to see for the $\mathrm{E}_{\mathrm{y}}$ amplitudes. The difference between the data from models with an anisotropic and with an isotropic target layer is very small in both $\mathrm{E}_{\mathrm{x}}$ and $\mathrm{E}_{\mathrm{y}}$ and the details cannot be readily discerned. These differences in the electric field between these two target types are typically on the same order of magnitude as the noise level.

In this first section of the paper we have chosen to examine the difference between responses from an isotropic target and an anisotropic model relative to the expected data noise level in order to more accurately access the level of sensitivity to the anisotropy. We have chosen the expression in equation 1 as sensitivity $\mathrm{s}_{\mathrm{E}}$ : 
$S_{E}=\frac{\left|E_{\text {aniso }}-E_{\text {iso }}\right|}{\left|E_{\text {noise }}\right|}$,

which is relative to the noise level. The sensitivity to the horizontal anisotropy is defined as the difference between field amplitudes (we use $\mathrm{E}_{\mathrm{x}}$ or $\mathrm{E}_{\mathrm{y}}$ ) from a model with an anisotropic resistive layer $\mathrm{E}_{\text {aniso, }}$, and the field amplitude from a model with an isotropic resistive layer model $\mathrm{E}_{\text {iso }}$ divided by a model of the expected noise measurement in order to quantify the sensitivity potentially obtainable in a commercial data set. A sensitivity $\mathrm{s}_{\mathrm{E}}$ value of 2 implies the difference between the anisotropic and isotropic electric field values is twice the noise value, $\mathrm{E}_{\text {noise, }}$ at that location. We define the noise as,

$$
E_{\text {noise }}=\sqrt{E_{\text {rel }}^{2}+E_{\text {rot }}^{2}+E_{\text {abs }}^{2}} \text {. }
$$

There are three contributions to the noise. The first contribution is a relative error $\left(\mathrm{E}_{\mathrm{rel}}\right)$ in the field amplitude expressed as a percentage of the amplitude. This can be due to systematic measurement or instrumental error and is assigned to be $5 \%$ in this study. The absolute error $\left(\mathrm{E}_{\mathrm{abs}}\right)$ is the transmitterreceiver system noise floor, which we take to be an optimistic $10^{-16} \mathrm{~V} / \mathrm{Am}^{2}$ in this study. The error due to rotation $\left(\mathrm{E}_{\mathrm{rot}}\right)$ specifically arises from the uncertainty in the receiver dipole orientation. If $\varphi$ is a small error in the rotation angle, $F_{x}$ and $F_{y}$ are the fields we measure, then the true fields $E_{x}, E_{y}$, can be given by the following matrix equation,

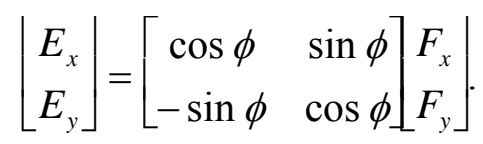

The error due to this rotation error is then $\Delta \mathrm{E}_{\mathrm{x}}=\mathrm{E}_{\mathrm{x}}-\mathrm{F}_{\mathrm{x}}$. With some manipulation it can be shown that, 


$$
\begin{aligned}
& \left|\Delta E_{x_{\text {rot }}}\right|=\sqrt{(\cos \phi-1)^{2}\left|E_{x}\right|^{2}+\sin ^{2} \phi\left|E_{y}\right|^{2}}, \\
& \left|\Delta E_{y_{\text {rot }}}\right|=\sqrt{(\cos \phi-1)^{2}\left|E_{y}\right|^{2}+\sin ^{2} \phi\left|E_{x}\right|^{2}} .
\end{aligned}
$$

The total error is then given by:

$$
\begin{aligned}
& \left|E_{x_{\text {noise }}}\right|=\sqrt{\alpha^{2}\left|E_{x}\right|^{2}+(\cos \phi-1)^{2}\left|E_{x}\right|^{2}+\sin ^{2} \phi\left|E_{y}\right|^{2}+E_{a b s}^{2}}, \\
& \left|E_{y_{\text {noise }}}\right|=\sqrt{\alpha^{2}\left|E_{y}\right|^{2}+(\cos \phi-1)^{2}\left|E_{y}\right|^{2}+\sin ^{2} \phi\left|E_{x}\right|^{2}+E_{a b s}^{2}} .
\end{aligned}
$$

Morten et al. (2009) calculates the rotation error in a similar manner for the inline data alone. In this study we used a fixed rotation uncertainty $\varphi$ of 5 degrees.

Figure 3 shows $\mathrm{E}_{\mathrm{x}}$ and $\mathrm{E}_{\mathrm{y}}$ field amplitudes and their corresponding sensitivity $\mathrm{s}_{\mathrm{E}}$ calculated at $0.5 \mathrm{~Hz}$ for model 2. For Figure 3 the anisotropic layer has $R_{\text {horz }}=1 \Omega \mathrm{m}$ and $R_{v e r t}=40 \Omega \mathrm{m}$, the isotropic layer has $\mathrm{R}_{\text {horz }}=\mathrm{R}_{\mathrm{vert}}=40 \Omega \mathrm{m}$. Figure 3a shows the $\mathrm{E}_{\mathrm{x}}$ amplitudes for inline and $6 \mathrm{~km}$ offline data, and Figure $3 \mathrm{~b}$ shows the $\mathrm{E}_{\mathrm{y}}$ amplitudes for $3 \mathrm{~km}$ and $6 \mathrm{~km}$ offline data. Due to the large amplitude range the differences between the two cases are difficult to see. When the sensitivities relative to the noise (Equation 1) are displayed in Figure $3 \mathrm{c}$ and $3 \mathrm{~d}$, the subtle differences are apparent. Both $\mathrm{E}_{\mathrm{x}}$ and $\mathrm{E}_{\mathrm{y}}$ components become more sensitive as the offline distance increases and $\mathrm{E}_{\mathrm{x}}$ is more sensitive at $6 \mathrm{~km}$ than $\mathrm{E}_{\mathrm{y}}$. At source receiver offsets much greater than $10 \mathrm{~km}$, the data becomes comparable to the noise level and the sensitivity goes to zero. Overall the inline data is above the noise floor to a few kilometers further distances than the offline data. 
One method to mitigate uncertainties in the receiver orientation is to decompose the data into the electromagnetic polarization ellipse parameters (e.g., Smith and Ward, 1974). The polarization ellipse can be constructed from the locus of the resultant electric field vector when combining the $E_{x}$ and $E_{y}$ electric field amplitudes and relative phases. $\mathrm{P}_{\max }$, which is the semi-major axis of the ellipse, is a more robust measure of the electric field since it is independent of the receiver orientation, it only depends on the amplitudes of the $\mathrm{E}_{\mathrm{x}}$ and $\mathrm{E}_{\mathrm{y}}$ components and their relative phase difference and is therefore not affected by absolute phase errors arising from unknown timing offsets between the source and receiver clocks. With a polarization ellipse decomposition, all of the orientation uncertainty is isolated into a single parameter, the ellipse orientation angle, which can be disregarded so that the interpretation focuses only on the $\mathrm{P}_{\max }$ data. However, it is worth noting that some conductivity information is lost when the ellipse orientation is ignored. For example, Key and Lockwood (2010) showed up to a 20-degree difference in the ellipse orientation for offline data when the seafloor conductivity changed only by a factor of two. With $\mathrm{P}_{\max }$, the sensitivity to the anisotropy now can be expressed by the sensitivity $\mathrm{s}_{\mathrm{p}}$ :

$S_{P}=\frac{\left|P_{\text {aniso }}-P_{\text {iso }}\right|}{\left|P_{\text {noise }}\right|}$

where $\mathrm{P}_{\text {noise }}$ is given by:

$P_{\text {noise }}=\sqrt{P_{r e l}^{2}+P_{a b s}^{2}}$.

$\mathrm{P}_{\text {rel }}$ is an assumed 5\% noise of the $\mathrm{P}_{\max }$ values for the anisotropic layer fields, and is due to systematic instrumental measurement errors. $\mathrm{P}_{\mathrm{abs}}$ is an absolute error floor taken to be $10^{-16} \mathrm{~V} / \mathrm{Am}^{2}$. Notice in this case there is no rotation error since we utilize both $\mathrm{x}$ and $\mathrm{y}$ components in the polarization calculation. A sensitivity $s_{p}$ value of 2 implies the difference between the anisotropic and isotropic $\mathrm{P}_{\max }$ values is twice the typical error value, $\mathrm{P}_{\text {noise, }}$ at that location. 
To visualize the location of highest sensitivity, Figure 4 displays the values $\mathrm{s}_{\mathrm{E}}$ and $\mathrm{s}_{\mathrm{p}}$ calculated for the entire survey area for models 1,2 and 3 . The plots also contain grey contour lines that mark the $10^{-15}$ $\mathrm{V} / \mathrm{Am}^{2}$ (inner) and the $10^{-16} \mathrm{~V} / \mathrm{Am}^{2}$ (outer) amplitude levels, where the $10^{-16} \mathrm{~V} / \mathrm{Am}^{2}$ contour is the noise floor used in sensitivity formulations shown earlier $\left(\mathrm{E}_{\mathrm{abs}}\right)$. The data located outside this contour are below the noise floor and hence would not be obtainable in a real survey. The rapid diminishing of sensitivity as the source receiver distance increases is due to the data reaching this noise floor. The white contour is where the sensitivity, $\mathrm{s}_{\mathrm{E}}$ or $\mathrm{s}_{\mathrm{p}}$ is equal to 1 and is where the difference between the anisotropic and isotropic fields or polarization parameters is equal to the noise level. The colored regions contained within the white contour are where the responses have sensitivity above the noise floor, and thus where it is possible to discriminate the subtle anisotropic signal.

The first column in Figure 4 shows the sensitivity using the $E_{x}$ field amplitude. At very short offsets the anisotropic sensitivity is zero due to the basic lack of sensitivity to the deep reservoir. The sensitivity increases moving away from the source in general due to the increased coupling with the reservoir, but there is a marked geometry aspect to the $\mathrm{E}_{\mathrm{x}}$ sensitivity. The purely inline and offline (broadside) regions have the highest sensitivity, but this diminishes rapidly where the $\mathrm{E}_{\mathrm{x}}$ amplitude drops to zero due to the basic dipole geometry (roughly along 45 degree azimuths). The maximum sensitivity value for models 1, 2 and 3 are found at offline distances of $7 \mathrm{~km}, 6 \mathrm{~km}$ and $6 \mathrm{~km}$, respectively along $\mathrm{x}=0$ $\mathrm{km}$, directly perpendicular to the x pointing dipole source. For models 1 and 2 the peak sensitivity value is around 7 and 4 . For model 3 where the target is at $3 \mathrm{~km}$ BML the sensitivity barely exceeds 1 for any source location.

The second column in Figure 4 shows the sensitivity to the $\mathrm{E}_{\mathrm{y}}$ field amplitude component. In this case 
the regions with sensitivity above 1 (above the noise level) all reside offline in patches away from the $\mathrm{x}$ and $\mathrm{y}$-axis, as expected given that $\mathrm{E}_{\mathrm{y}}$ component has zero amplitude along the $\mathrm{x}$ and $\mathrm{y}$ axes due to the dipole geometry. The sensitivity tends to increase moving further offline away from the source axis. For model 1 where the target is at $1 \mathrm{~km}$ depth, the region of high sensitivity covers a greater spatial extent for $\mathrm{E}_{\mathrm{y}}$ data compared to the $\mathrm{E}_{\mathrm{x}}$ data, however the peak values are lower. Overall the $\mathrm{E}_{\mathrm{y}}$ component sensitivities are lower than $\mathrm{E}_{\mathrm{x}}$ and do not exceed value of 3, even for the shallowest target.

The $\mathrm{P}_{\max }$ sensitivities for models 1, 2, 3 are shown in the final column. Since $\mathrm{P}_{\max }$ is computed using both $E_{x}$ and $E_{y}$, its sensitivity has a shape that is a combination of the sensitive regions of the individual components. The highest sensitivity to the anisotropy is consistently in the furthest offline data within the noise floor boundaries. There is, to a lesser extent, sensitivity in the inline regions that are lower and lie further from the source compared to the offline data, giving the sensitivity an ellipsoidal shape that is elongated in the inline (x) direction. Model 1 has a peak sensitivity of around 7.5, model 2 the peak is around 4. Model 3 does not have sensitivity above the noise floor, despite $\mathrm{P}_{\max }$ being insensitive to the rotation error.

Figure 5 shows the peak sensitivities, $\mathrm{s}_{\mathrm{E}}$, over the entire survey area as a function of source frequency and a range of target layer anisotropy values created by increasing the $\mathrm{R}_{\mathrm{horz}}$ in the layer from $1 \Omega \mathrm{m}$ to $20 \Omega \mathrm{m}\left(\mathrm{R}_{\mathrm{vert}}=\Omega \mathrm{m}\right)$. This study was done for model 2 where the target layer is $2 \mathrm{~km}$ BML. The largest sensitivity above the noise floor is in the range of $0.25-1 \mathrm{~Hz}$ with a peak at $0.5 \mathrm{~Hz}$. The sensitivity rapidly decreases for both higher and lower frequencies. As $\mathrm{R}_{\text {horz }}$ increases and the layer becomes less anisotropic, the sensitivity to anisotropy decreases, as expected (by definition it goes to zero for a perfect isotropic layer). Figure 5 shows that even for a very modest increase in $\mathrm{R}_{\text {horz }}$ from 1 to $5 \Omega \mathrm{m}$ ( $R_{\text {horz }}$ is comparable to the background $R_{\text {horz }}$ ) the $E_{x}$ sensitivity changes from greater than 4 to less than 
1 at $0.5 \mathrm{~Hz}$. When $\mathrm{R}_{\text {horz }}$ is $5 \Omega \mathrm{m}$ the difference between the isotropic and anisotropic fields would be difficult to detect without further stacking of the data, or without future instrumental improvements to lower the source-receive noise floor. The $\mathrm{E}_{\mathrm{y}}$ sensitivities show a similar pattern but overall have much lower sensitivity than $E_{x}$.

Figure 6 shows the sensitivity values using at the same definition and color scales as Figure 4 for a 3D slab example, described in Table 1 . Note that in this study the depth of the target is held fixed at $2 \mathrm{~km}$ depth and the three rows represent scenarios where the source is placed at the origin, at $4 \mathrm{~km}$ and $6 \mathrm{~km}$ from the origin along the y axis. Overall the sensitivities are significantly less than for the layered model at the same depth of $2 \mathrm{~km}$ (second row in Figure 4). The $\mathrm{E}_{\mathrm{y}}$ component alone is now insensitive to the anisotropy, suggesting that the $\mathrm{E}_{\mathrm{y}}$ sensitivity in the 1D studies depends on the large lateral extent of the target. When the source is directly over the target (shown in the top row), the sensitivity is above the noise level only for the offline $\mathrm{E}_{\mathrm{x}}$ responses. The middle row shows the target is coupled optimally when the source is located $4 \mathrm{~km}$ from the target center, as illustrated by the peak sensitivity of around 3. The sensitive data is localized to a small region located $6-8 \mathrm{~km}$ offline. The bottom row shows the data collected when the source is at $6 \mathrm{~km}$ from the origin, again there is sensitivity offline but it is lower than the optimally coupled $4 \mathrm{~km}$ source position. The $\mathrm{P}_{\max }$ sensitivities for each case tend to reflect the $E_{\mathrm{X}}$ sensitivities since the $\mathrm{E}_{\mathrm{x}}$ component is the main contributor.

\section{STOCHASTIC INVERSIONS}

In this section we use the $1 \mathrm{D}$ stochastic inversion algorithm developed by Chen et al. (2007) to determine the ability to resolve the anisotropic parameters of a resistive target in model 2 in an inverse 
sense. We assume that the location and thickness of the reservoir target can be determined from seismic data or other information. We estimate $\mathrm{R}_{\text {vert }}$ and anisotropy ratio, $\lambda$. The resistivity of seawater, the overburden and the bedrock are also considered unknowns. We estimate parameters using data along two survey lines, inline $(\mathrm{y}=0 \mathrm{~m})$ and offline $(\mathrm{y}=6 \mathrm{~km})$, for a model with an isotropic target $\left(\mathrm{R}_{\text {horz }}=\mathrm{R}_{\text {vert }}=\right.$ $40 \Omega \mathrm{m})$ and an anisotropic target $\left(\mathrm{R}_{\text {horz }}=1 \Omega \mathrm{m}\right.$ and $\left.\mathrm{R}_{\text {vert }}=40 \Omega \mathrm{m}\right)$. We use the same priors for the two cases and assume that $R_{\text {vert }}$ is uniformly distributed on $(1,200) \Omega m$, and the ratio $R_{\text {vert }} / R_{\text {horz }}$ is uniformly distributed on $(1,100)$. The prior distributions of seawater, overburden, and bedrock resistivity are uniformly distributed on $(0.1,1.0) \Omega \mathrm{m},(1,10) \Omega \mathrm{m}$, and $(1,10) \Omega \mathrm{m}$, respectively. While this 1D example is a simplified model with a very small number of parameters (five) and is not representative of the resolution that could be achieved in real-world inversions of field data without perfect a priori knowledge of the layer boundaries; it merely serves to illustrate the relative differences in resolution of $\mathrm{R}_{\text {horz }}$ versus $\mathrm{R}_{\text {vert }}$ for different data types (i.e. inline and offline data).

Markov chain Monte Carlo methods are used to explore the joint posterior distribution of unknowns. A hybrid sampling strategy described in Chen et al. (2007) is used. The sampling methods include: (1) single variable Metropolis-Hastings methods (SMH) (Metropolis et al., 1954; Hastings et al., 1970), (2) multivariate Metropolis-Hastings methods (MMH), (3) single variable slice sampling methods (SSS) (Neal, 2003), and (4) multivariate slice sampling methods (MSS). At each iteration we randomly select one of above methods for updating. We start from five different sets of initial values, which are the lower bounds, upper bounds, median, $25 \%$ quantile, and $75 \%$ quantiles of the prior bounds. We use the potential scale reduction factor (PSRF) to monitor the convergence of the five chains using the method by Brooks and Gelman (1998), which is a measure of the between-chain variability relative to the within-chain variability. With that approach, if the scale reduction score is less than 1.2, the Markov chain is considered converged; otherwise, more runs are needed. 
The stochastic inversion was run with 5\% Gaussian noise added to the synthetic data. Figures $7 \mathrm{a}$ and $7 \mathrm{~b}$ show the parameter PDFs obtained for the horizontal and vertical resistivity for the isotropic target model. Based on the forward modeling results we would expect the inline data to have the highest sensitivity to $\mathrm{R}_{\mathrm{vert}}$ and the offline data to have the highest sensitivity to $\mathrm{R}_{\text {horz, }}$ which is consistent with the mode and standard deviations of these parameters listed in Table 2. The mode of both the inline and offline PDFs are within $1 \%$ of $\mathrm{R}_{\mathrm{vert}}$, as expected. Conversely, for $\mathrm{R}_{\mathrm{horz}}$, the offline mode is within $15 \%$ while the mode of the inline data is $38 \%$ too low. This result indicates that there is information about $\mathrm{R}_{\text {horz }}$ in the inline since the PDF clearly indicates it is resistive. The larger parameter standard deviations (width of the PDFs) for $\mathrm{R}_{\text {horz }}$ compared to $\mathrm{R}_{\mathrm{vert}}$ reflects the lower sensitivity to $\mathrm{R}_{\mathrm{horz}}$ which was seen in the forward modeling studies.

The parameter PDFs for the anisotropic target layer are shown in Figure $8 \mathrm{a}$ and $8 \mathrm{~b}$. The results for $\mathrm{R}_{\mathrm{vert}}$ are consistent with that of the isotropic case, with the PDFs for both inline and offline data within $1 \%$ of the true values. The PDFs for $\mathrm{R}_{\text {horz }}$ show that both the inline and offline data resolve the $\mathrm{R}_{\text {horz }}$ better than the isotropic case. The mode is closer to the true value and the standard deviation smaller using the offline data compared to the inline.

The stochastic inversions lead to two main conclusions; firstly that $\mathrm{R}_{\mathrm{vert}}$ of the $1 \mathrm{D}$ target layer is well resolved by both inline and offline data, and secondly that the anisotropic layer $\mathrm{R}_{\text {horz }}$ of $1 \Omega \mathrm{m}$ is much better resolved than the more resistive isotropic layer $\mathrm{R}_{\text {horz }}$ of $40 \Omega \mathrm{m}$. To further explore how the ability to resolve $R_{\text {horz }}$ may be affected by the value of $R_{\text {horz }}$ in the target layer, data was simulated for anisotropic models and a reference model with the same geometry as model 2 . In this section the reference model target layer $R_{\text {horz }}$ is equal to the basement $R_{\text {horz }}$ of $4 \Omega \mathrm{m}$ and $R_{v e r t}$ is $40 \Omega \mathrm{m}$. The 
anisotropy is varied in the target layer by fixing $R_{\text {vert }}$ at $40 \Omega \mathrm{m}$, varying $R_{\text {horz, }} 1-20 \Omega \mathrm{m}$, which ranges from less than, to greater than $R_{\text {horz }}$ of the reference model . For each model a percentage difference is calculated, to determine how sensitive the electric field response is to the value of $\mathrm{R}_{\text {horz }}$ compared to the background value. Here we are concerned with the variation in field response as $R_{\text {horz }}$ is varied and we are unconcerned with the measurement noise. The equation

$$
S_{P D}=\frac{\left|E_{\text {aniso }}-E_{\text {ref }}\right|}{\left|E_{\text {ref }}\right|} \times 100,
$$

where $E_{\text {aniso }}$ is the $E_{x}$ field for the anisotropic reservoir and $E_{\text {ref }}$ is the $E_{x}$ field from the reference model is used. This percentage difference, $\mathrm{SPD}_{\mathrm{PD}}$, versus the target layer $\mathrm{R}_{\text {horz }}$ is plotted in Figure $9 \mathrm{a}$ and $9 \mathrm{~b}$ for inline and $6 \mathrm{~km}$ offline data respectively. For the inline and offline cases there is a large increase in percentage difference as $R_{\text {horz }}$ becomes more conductive than the reference/background value. There is a much smaller increase as $R_{\text {horz }}$ becomes more resistive than the background. In both cases all data below the noise floor is removed which explains why the offline figure (Figure 9b), despite being more sensitive to the anisotropy, only contains the two lowest frequencies. The large gradient as $\mathrm{R}_{\text {horz }}$ is made more conductive compared to more resistive explains why the stochastic inversion resolves the conductive $R_{\text {horz }}$ better than the resistive $R_{\text {horz }}$. When $R_{\text {horz }}$ is more resistive than $4 \Omega m$ (the background), the change in electric field response decreases. This effect appears similar to the saturation effect observed for the magnetotelluric method, where the magnetotelluric response can be shown to saturate once the resistivity contrast for a resistive layer reaches a certain limit (typically about a factor of 10 more resistive than the background).

To gain further physical insight into this saturation behavior, here we look at the field and current density sections in the $\mathrm{x}-\mathrm{z}$ plane through the target layer for model 2. Figure 10 shows the E field and 
current density polarization ellipses in the $\mathrm{x}-\mathrm{z}$ axis $(\mathrm{y}=0$, inline) computed using a $2 \mathrm{D}$ adaptive finite element code (Key and Ovall, 2011) after minor modifications to handle anisotropy. The background color is the $\mathrm{P}_{\max }$ amplitude for the anisotropic reservoir case; the white polarization ellipses are for the anisotropic reservoir, the black are the isotropic reservoir. Figure 10a shows the electric field polarizations. However, it is difficult to see the anisotropic ellipses (white) underneath the isotropic data ellipses (black), illustrating that the electric field polarization is nearly identical for the anisotropic and isotropic target cases. Inside the reservoir layer at 4.5 to $4.6 \mathrm{~km}$ depth, both anisotropic and isotropic fields are vertically polarized, as expected from the electromagnetic version of Snell's law considered for the TM mode at the boundary between a conductor and resistor. In Figure $10 \mathrm{~b}$ to $\mathrm{d}$ we show the electric current density polarizations, computed by multiplying the electric fields by the anisotropic conductivity. Now we can see that the current polarizations have a significantly different shape in the reservoir layer, depending on the horizontal resistivity. The isotropic target is predominantly vertically polarized, whereas the anisotropic target has a large horizontal component and the ellipses are more horizontally polarized, especially at short offsets. At farther offsets there is still a large horizontal current density component compared to the isotropic target but at longer offsets the vertical current density component increases fairly rapidly, making the ellipses vertically polarized. The difference is most pronounced when $R_{\text {horz }}$ is $1 \Omega \mathrm{m}$ (Figure $1 \mathrm{~b}$ ). As $\mathrm{R}_{\text {horz }}$ increases the current density ellipses tend to more closely resemble the isotropic case with vertical polarization inside the reservoir. Figure 11 shows the horizontal and vertical components of the current density at the center of the target layer at $3 \mathrm{~km}$ offset from the source ( $x=3 \mathrm{~km}, \mathrm{z}=4.55 \mathrm{~km}$ ) for a range of $\mathrm{R}_{\text {horz }}$ with $\mathrm{R}_{\text {vert }}$ fixed at $40 \Omega \mathrm{m}$. As one would expect, the vertical current density stays constant as $R_{\text {horz }}$ increases since the vertical conductivity remains unchanged. The horizontal current density decreases in proportion to $1 / R_{\text {horz }}$, as expected from Ohm's law. When $R_{\text {horz }}$ is less than $2.5 \Omega \mathrm{m}$, the horizontal current density is larger than the vertical density, whereas at greater values of $R_{\text {horz }}$ the horizontal current density is far 
below the vertical density. It is at this point that the field and current are dominantly vertical.

Therefore, we can now explain the saturation effect shown in Figure 9 as arising from the transition from horizontally dominated current in the reservoir to a vertically dominated current when $\mathrm{R}_{\text {horz }}$ becomes greater than $2.5 \Omega \mathrm{m}$. Once the polarization is dominantly vertical, sensitivity to the horizontal conductivity has saturated and the sensitivity to anisotropy in a target is low.

\section{D GRADIENT BASED ANISOTROPIC INVERSION}

We have seen from the first two sections, the sensitivity analysis and the stochastic inversions, that there is information in the inline and offline electric fields that provides high sensitivity to the vertical electrical resistivity and to a lesser extent the horizontal electrical resistivity of the target layer or slab. In particular we see that when the horizontal resistivity is conductive relative to the vertical and background resistivity, the 1D stochastic inversion shows it can be well resolved. However, once the horizontal resistivity becomes resistive relative to the background it is less well resolved, although it is found to be more resistive than the background.

Unfortunately, most exploration scenarios are not well modeled by $1 \mathrm{D}$ and require at least $2 \mathrm{D}$ and often 3D inversion of the field data to accurately model potential reservoirs and their surrounding structures. While any study of resolution using a specific inversion code is always open to the criticism that results are specific to the code used, we feel it is still instructive. Additionally, we are using a code that is in wide use throughout industry, the LBNL “EM3D_GEO” code (Newman and Alumbaugh, 1997; Newman and Hoversten, 2000, Newman et al. 2010). 
For the 3D inversion studies we use the 3D slab model shown in Figure 1b, where the target layer is a 5 $\mathrm{km} \times 5 \mathrm{~km}$ slab, $100 \mathrm{~m}$ thick at a depth of $2 \mathrm{~km} \mathrm{BML}$, and with the same vertical and horizontal resistivity as used for the $1 \mathrm{D}$ model. Frequencies of $0.125,0.25,0.5,1.0$ and $2.0 \mathrm{~Hz}$ are used. The model inversion grid is discretized at 150,150 , and $100 \mathrm{~m}$ in $\mathrm{x}, \mathrm{y}$ and $\mathrm{z}$ respectively. The inversion used a non-linear transformation of the model parameters to bound resistivity throughout the model to between 0.5 to $100 \Omega \mathrm{m}$, unless otherwise noted. An array of 25 receivers is laid out symmetrically over the target with $2 \mathrm{~km}$ separations in both $\mathrm{x}$ and $\mathrm{y}$. Source tow-lines of up to $15 \mathrm{~km}$ are run over the array with inline and offline components in both $\mathrm{x}$ and $\mathrm{y}$. The maximum offline distance is $8 \mathrm{~km}$ in the first examples. For the $8 \mathrm{~km}$ offsets we assumed a noise floor of $10^{-16} \mathrm{~V} / \mathrm{Am}^{2}$ to allow sufficient number of data at the $8 \mathrm{~km}$ offsets. A maximum offline distance of $4 \mathrm{~km}$ with a $10^{-15} \mathrm{~V} / \mathrm{Am}^{2}$ noise floor is also considered, reflecting what we normally consider usable data from field operations. The choice of how much offline data to use varies with circumstances, some operations use data to larger offline distances, but we have found that it is generally very noisy, more so than our simple noise model (\% of amplitude plus rotation error) accounts for. We will not speculate on the source of this addition error as a function of offline distance but simply note its existence. Synthetic data was contaminated by Gaussian noise with standard deviation set to $2 \%$ of the amplitude of the electric field. This value was chosen to match the error level used by Abubakar et al. (2010). All inversions shown are for the iteration with RMS misfit just below 1.0. The inversion started from the true background model without the anisotropic target present. This can be considered a best-case scenario; our experience indicates that the determination of the position and absolute resistivity of targets worsens as the accuracy of the background (starting) model decreases. 
We first consider the inversion of the isotropic target where $R_{\text {horz }}$ and $R_{\text {vert }}$ is $40 \Omega \mathrm{m}$. Figure 12 shows vertical cross sections through the center of the target body $(y=0)$. Figure 13 shows plan views of the $\mathrm{R}_{\mathrm{vert}}$ and $\mathrm{R}_{\text {horz }}$ at the depth slices of maximum resistivity. We see the spatial location of the target is well defined (Figure 13a) with the maximum $\mathrm{R}_{\mathrm{vert}}$ of $10.5 \Omega \mathrm{m}$ as compared to the true value of $40 \Omega \mathrm{m}$ at a depth $100 \mathrm{~m}$ deeper than the target. $\mathrm{R}_{\text {horz }}$ is much less well resolved. The maximum $\mathrm{R}_{\text {horz }}$ is $2.1 \Omega \mathrm{m}$ at a depth of 3900-4000 m compared to the true value of $40 \Omega \mathrm{m}$. While the inverted $\mathrm{R}_{\text {horz }}$ shows an indication that the target has higher $\mathrm{R}_{\text {horz }}$ than the background it is very slight. In a true $3 \mathrm{D}$ environment with variable structure and background resistivity this variation would not be considered significant. In this case we would not be able to reliably indicate that the target was either isotropic or anisotropic.

The vertical positioning of the anomalous $\mathrm{R}_{\mathrm{vert}}$ is enhanced by its being at the contact between conductive and more resistive material at $2 \mathrm{~km}$ BML. Studies using EM3D_GEO have shown that in a half-space background with no constraints (either removing smoothing across boundaries, or imposing bounds on allowable resistivity in certain regions) the resistive target would focus approximately $10 \%$ $(200 \mathrm{~m})$ too shallow. The maximum resistivity will always be less than the true value with the difference increasing with depth of the target. The lower resistivity is somewhat compensated in the inversion with an increase in the thickness so that the reservoir resistivity-thickness product is preserved, as is also typically seen in 1D inversions (e.g., Key 2009; Brown et al, in review).

Next we consider the anisotropic 3D target where $R_{\text {vert }}=40 \Omega \mathrm{m}$ and $R_{\text {horz }}=1.0 \Omega \mathrm{m}$. Figure 14 shows the vertical cross sections and Figure 15 the horizontal depth slices. The reconstruction of $\mathrm{R}_{\mathrm{vert}}$ in the vertical cross sections is not significantly different compared to the isotropic case. The maximum resistivity of $12.5 \Omega \mathrm{m}$ occurs $100 \mathrm{~m}$ too deep. The inversion of $\mathrm{R}_{\text {horz }}$ (Figure $14 \mathrm{~b}$ ) has produced a region of lower resistivity, $1.5 \Omega \mathrm{m}$ compared to the true value of $1.0 \Omega \mathrm{m}$, which is $200 \mathrm{~m}$ too shallow. 
The spatial image of both $R_{v e r t}$ and $R_{\text {horz }}$ (Figure 15) shows good agreement with the lateral extent of the slab. In this case where the anisotropic target has more conductive $\mathrm{R}_{\text {horz, }}$, the lateral resolution of $R_{\text {horz }}$ is much better than in the isotropic case (compare Figure $15 b$ with $11 b$ ). In these examples the $3 D$ inversion is insensitive to anisotropy.

Reducing the maximum offline separation from $8 \mathrm{~km}$ with a $10^{-16} \mathrm{~V} / \mathrm{Am}^{2}$ noise floor to $4 \mathrm{~km}$ with a $10^{-15} \mathrm{~V} / \mathrm{Am}^{2}$ noise floor has only a minor effect on inversion results. The inverted $\mathrm{R}_{\mathrm{vert}}$ for the anisotropic model produces $15 \Omega \mathrm{m}$ as a maximum resistivity and this occurs at the true depth of 4500 $4600 \mathrm{~m}$. This is compared to $12 \Omega \mathrm{m}$ and $100 \mathrm{~m}$ too deep for the $8 \mathrm{~km}$ offset inversion of the anisotropic model. The isotropic model results are almost identical.

While a complete study of the effects of inversion constraints is beyond the scope of this paper, one illustration is worth considering. In practice, prior knowledge including wells, seismic data and geology of the basin provide additional constraints that can be used in inversion. Figure 16 shows the inversion results for the isotropic reservoir model with maximum $4 \mathrm{~km}$ offset data and $10^{-15} \mathrm{~V} / \mathrm{Am}^{2}$ noise floor when the upper bound on allowed resistivity $\left(R_{\text {horz }}\right.$ and $\left.R_{v e r t}\right)$ in the overburden is changed from 100 to $10 \Omega \mathrm{m}$ and the inversion's roughness penalty (i.e., smoothing) is removed across the top and base of the target slab. Now even with the reduced amount of data, reflecting a more likely field case, the maximum inverted $\mathrm{R}_{\text {vert }}$ reaches $33 \Omega \mathrm{m}$ compared to $12.5 \Omega \mathrm{m}$ in the less constrained case (Figure 12). The added constraints have not however improved the resolution of $\mathrm{R}_{\text {horz }}$.

As was seen in the 1D inversion results of the isotropic versus anisotropic layer, the inversion is more sensitive to conductive $R_{\text {horz }}$ than resistive $R_{\text {horz. }}$. However, the ability to resolve $R_{\text {vert }}$ and $R_{\text {horz }}$ for a $3 D$ target is far worse than for a 1D layer. While one can always argue that a different algorithm may 
produce improved results, the models found by this algorithm fit the data to better than $2 \%$ and clearly demonstrates that there is large non-uniqueness in the 3D model space. The 3D inversions were also run for the equivalent of model 1, where the slab was moved to $1 \mathrm{~km}$ BML. In these tests (not shown) the extreme values of the inverted resistivity came closer to the true values but were still not nearly as accurate as 1D examples with the target layer $1 \mathrm{~km}$ BML.

\section{CONCLUSIONS}

We conducted a suite of simulation studies to understand the sensitivity and resolution capabilities of marine CSEM for detecting electrical anisotropy within a reservoir. Since the horizontal resistivity can help distinguish between an anisotropic shale from an isotropic reservoir, our studies have paid particular attention to the sensitivity to horizontal resistivity. The $1 \mathrm{D}$ sensitivity studies showed that deep water (2.5 km water depth) CSEM surveys using a HED source are relatively insensitive to modest anisotropy in a reservoir target. This is especially true when the target horizontal resistivity is larger than the background horizontal resistivity. By using a somewhat realistic noise model that includes relative and absolute noise sources, as well as uncertainty in the receiver orientation, we showed that the areas of low but detectable sensitivity are predominantly localized offline at distances of 3-8 $\mathrm{km}$. The sensitivity to the reservoir diminishes rapidly as the reservoir depth increases and as the anisotropy ratio decreases. The sensitivity also decreases rapidly at higher and lower source frequencies than $0.5 \mathrm{~Hz}$. Well constrained stochastic 1D inversion results showed that there is sensitivity to the horizontal resistivity in both inline data and to a greater extent, in the offline data. Stochastic inversion results and the deterministic 3D inversion results highlight an important consideration in resolving transverse anisotropy in a target. That is, as the horizontal resistivity decreases below the background 
resistivity the data sensitivity to the anisotropy increases. If the horizontal resistivity is greater than or comparable to the background resistivity the data is far less sensitive to the anisotropy. Although the $3 \mathrm{D}$ inversion is unable to resolve the correct horizontal and vertical resistivity, when the target horizontal resistivity is more conductive than the background the resolution is slightly better. In general for the 3D inversion (which far is less constrained than the 1D stochastic inversion) the reservoir vertical resistivity is better resolved than horizontal resistivity, particularly when the horizontal resistivity of the target is greater than or equal to the background resistivity. Examination of the current density polarization within the reservoir shows that the decreasing sensitivity to horizontal resistivity and hence to anisotropy in the reservoir is due to the reservoir current becoming vertically dominant as horizontal resistivity increases, resulting in a saturation of the sensitivity to horizontal resistivity.

Although the HED source has sensitivity to the target horizontal resistivity, the sensitivity to the vertical resistivity is greater than that of the horizontal resistivity. We conclude that to be able to discriminate modest anisotropy in a deep water target $(2.5 \mathrm{~km})$ using CSEM either a new source configuration or a combination of HED and HMD sources may be required.

\section{ACKNOWLEGMENTS}

We are grateful to Chevron Energy Technology Company for support and permission to publish this work. Brown and Key acknowledge funding support from the Seafloor Electromagnetic Methods Consortium at Scripps Institution of Oceanography. This work was also partially supported by the U.S. 
Department of Energy and LBNL under Contract No. DE-AC02-05CH11231.

\section{REFERENCES}

Abubakar, A., 2010, Sensitivity study of multi-sources receivers CSEM data for TI- anisotropy medium using 2.5D forward and inversion algorithm: Presented at the 72nd Annual EAGE Conference.

Al-Garni, M., and M. Everett, 2003, The paradox of anisotropy in electromagnetic loop- loop responses over a uniaxial half-space: Geophysics, 68, 892-899.

Anderson, B., I., Bryant, M., Luling, B., Spies, and K., Helbig, 1994, Oilfield anisotropy--its origins and electrical characteristics: Schlumberger Oilfield Review, 6.4, 48-56.

Barber T., B. Anderson, A. Abubakar, T. Broussard, K. Chen, S. Davydycheva, V. Druskin, T.Habashy, D. Homan, G. Minerbo, R. Rosthal, R. Schlein, H. Wang, Determining formation resistivity anisotropy in the presence of invasion: SPE Annual Technical Conference and Exhibition, 26-29 September 2004, Houston, Texas

Chen, J., G. Hoversten, D. Vasco, Y. Rubin, and Z. Hou, 2007, A Bayesian model for gas saturation estimation using marine seismic AVA and CSEM data: Geophysics, 72, WA85-95.

Constable, S., and C. J. Weiss, 2006, Mapping thin resistors and hydrocarbons with marine EM 
methods: Insights from 1D modeling: Geophysics, 71(2), G43-G51.

Ellis, M., H., M. C. Sinha, T. A. Minshull, J., Sothcott,, A., Best, 2010, An anisotropic model for the electrical resistivity of two-phase geologic materials: Geophysics, 75, E161

Everett, M., and S. Constable, 1999, Electric dipole fields over an anisotropic seafloor: theory and application to the structure of 40 Ma Pacific Ocean lithosphere: Geophysical Journal International, 136, 41-56.

Hoversten, G. M., T. Røsten, K. Hokstad, D. Alumbaugh, S. Horne, and G. A. Newman, 2006, Integration of multiple electromagnetic imaging and inversion techniques for prospect evaluation: SEG Technical Program Expanded Abstracts, 25, 719-723.

Key, K., 2009, 1D inversion of multicomponent, multifrequency marine CSEM data: Methodology and synthetic studies for resolving thin resistive layers, Geophysics, 74(2), F9-F20.

Key, K., and A. Lockwood, 2010, Determining the orientation of marine CSEM receivers using orthogonal procrustes rotation analysis, Geophysics, 75(3), F63-F70

Key, K. and J. Ovall, 2011, A parallel goal-oriented adaptive finite element method for 2.5D electromagnetic modeling, accepted Geophysical Journal International, 186, 137-154.

Klein, J. D., 1996, Saturation effects on electrical anisotropy: The Log Analysis, 37, 47-49. 
Klein, J.D., Martin, P.R., Allen, D.F., 1997, The Log Analyst, May-June, 25 - 36.

Le Masne, D., and G. Vasseur, 1981, Electromagnetic field of sources at the surface of a homogeneous conducting halfspace with horizontal anisotropy: Application to Fissured Media: Geophysical Prospecting, 29, 803-821.

Li, Y., and S. Dai, 2011, Finite element modeling of marine controlled-source electromagnetic responses in two-dimensional dipping anisotropic conductivity structures, Geophysical Journal International. 185, 622-636.

Lu, X., and D. Alumbaugh, 2001, Three-dimensional sensitivity analysis of induction logging in anisotropic media: Petrophysics, 42, 566-579.

Lu, X., and C. Xia, 2007, Understanding anisotropy in marine csem data: 79th Annual International Meeting, SEG, Expanded Abstracts 26, 633-637

Mansour al Garni and M. E. Everett, 2003, The Paradox of anisotropy in electromagnetic loop-loop responses over a uniaxial half-space, Geophysics, 68, 892-899

Morten, J . F., A. K. Bjorke, T. Storen, 2009, CSEM data uncertainty analysis for 3D inversion: SEG, Expanded Abstracts, 28, 724-728 
Newman, G. A., Alumbaugh, D. L, 1997, Three-dimensional massively parallel electromagnetic inversion - I Theory: Geophys. J. Int., 128, 345-354.

Newman, G. A., Hoversten, G. M., 2000, Solution strategies for two- and three-dimensional electromagnetic inverse problems: Inverse Problems, 16, 1357-1375.

Newman, G. A., Commer, M., Carazzone, J. J., 2010, Imaging CSEM data in the presence of electrical anisotropy: Geophysics, 75, F51-F61.

Orange, A., K. Key, and S. Constable, 2009, The feasibility of reservoir monitoring using time-lapse marine CSEM: Geophysics, 74, F21-F29.

Ramananjaona, C., L. MacGregor and D. Andréis, 2010, Sensitivity and inversion of marine electromagnetic data in a vertically anisotropic stratified earth: Geophysical Prospecting, 59, 341-360

Smith, B. D., and S. H. Ward, 1974, Computation of polarization ellipse parameters: Geophysics, 39, 867-869.

Tompkins, M., R. Weaver, and L. MacGregor, 2004, Effects of vertical anisotropy on marine active source electromagnetic data and inversions: Presented at the 66th Annual Conference and Exhibition, EAGE. 
Tompkins, M., 2005, The role of vertical anisotropy in interpreting marine controlled-source electromagnetic data: 75th Annual International Meeting, SEG, Technical Program Expanded Abstracts, 24, 514-517

Weidelt, P., 2007, Guided waves in marine CSEM: Geophysical Journal International, 171, 153-176. 


\section{LIST OF TABLES}

1 Table of synthetic models used for forward modeling

2 Table of mode and standard deviations for target layer in model 2 from stochastic inversion with $5 \%$ noise level

\begin{tabular}{|c|c|c|c|}
\hline & $\begin{array}{c}\text { Target Depth } \\
\text { BML }\end{array}$ & $\begin{array}{c}\text { Anisotropic } \\
\mathbf{R}_{\text {vert }}: \mathbf{R}_{\text {horz }}\end{array}$ & $\begin{array}{c}\text { Isotropic } \\
\mathbf{R}_{\text {vert }}: \mathbf{R}_{\text {horz }}\end{array}$ \\
\hline Model 1 & $1 \mathrm{~km}$ & $40: 1$ & $40: 40$ \\
\hline Model 2 & $2 \mathrm{~km}$ & $40: 1,40: 2.5,40: 5,40: 10,40: 20$ & $40: 40$ \\
\hline Model 3 & $3 \mathrm{~km}$ & $40: 1$ & $40: 40$ \\
\hline 3D Slab & $2 \mathrm{~km}$ & $40: 1$ & $40: 40$ \\
\hline
\end{tabular}

Table 1: Table of synthetic models used for forward modeling 


\begin{tabular}{|c|c|c|c|c|c|c|c|c|}
\hline & \multicolumn{4}{|c|}{ Isotropic Target } & \multicolumn{4}{|c|}{ Anisotropic Target } \\
\hline & \multicolumn{2}{|c|}{ Inline } & \multicolumn{2}{|c|}{ Offline } & \multicolumn{2}{|c|}{ Inline } & \multicolumn{2}{|c|}{ Offline } \\
\hline & $\mathbf{R}_{\text {vert }}$ & $\mathbf{R}_{\text {horz }}$ & $\mathbf{R}_{\text {vert }}$ & $\mathbf{R}_{\text {horz }}$ & $\mathbf{R}_{\text {vert }}$ & $\mathbf{R}_{\text {horz }}$ & $\mathbf{R}_{\text {vert }}$ & $\mathbf{R}_{\text {horz }}$ \\
\hline Value & 40 & 40 & 40 & 40 & 40 & 1 & 40 & 1 \\
\hline Mode & 40.12 & 25.41 & 40.22 & 34.35 & 40.18 & 1.03 & 40.17 & 1.00 \\
\hline StD & 0.105 & 67.1 & 0.170 & 49.2 & 0.104 & 0.055 & 0.216 & 0.019 \\
\hline
\end{tabular}

Table 2: Table of mode and standard deviations for target layer parameters in model 2 from stochastic inversions 


\section{LIST OF FIGURES}

Figure 1: (a) Models 1, 2, 3 are shown. The $100 \mathrm{~m}$ anisotropic target layer has variable $\mathrm{R}_{\text {horz }}$ and fixed $R_{v e r t}=40 \Omega \mathrm{m}$, the reference model has an isotropic layer with $R_{\text {horz }}=R_{\text {vert }}=40 \Omega \mathrm{m}$. (b) $3 \mathrm{D}$ slab model consists of a $100 \mathrm{~m}$ thick $5 \mathrm{~km}$ by $5 \mathrm{~km}$ slab with variable $\mathrm{R}_{\text {horz }}$ and fixed $\mathrm{R}_{\mathrm{vert}}=40$ $\Omega \mathrm{m}$, the reference 3D model has an isotropic slab with horizontal and vertical resistivity $\mathrm{R}_{\text {horz }}=$ $\mathrm{R}_{\mathrm{vert}}=40 \Omega \mathrm{m}$.

Figure 2: $E_{x}$ and $E_{y}$ field amplitudes at $0.5 \mathrm{~Hz}$ for model 2. The $E_{x}$ data is inline (a), the $E_{y}$ data is $3 \mathrm{~km}$ offline, (b) three variations of model 2 shown: with no target layer, an isotropic target layer and an anisotropic target layer.

Figure 3: $E_{x}$ and $E_{y}$ amplitudes and sensitivities at $0.5 \mathrm{~Hz}$. (a) shows $E_{x}$ amplitudes for inline and $6 \mathrm{~km}$ offline data for the isotropic $\left(\mathrm{R}_{\text {horz }}=\mathrm{R}_{\mathrm{vert}}=40 \Omega \mathrm{m}\right)$ and anisotropic layer $\left(\mathrm{R}_{\text {horz }}=1 \Omega \mathrm{m}\right.$ and $\mathrm{R}_{\mathrm{vert}}=40 \Omega \mathrm{m}$ ), shown as solid and dash lines respectively. $\mathrm{E}_{\text {noise }}$ is shown as the shaded region above and below each line (darker shading is isotropic field noise). The corresponding sensitivities (as defined in Equation 1) are plotted in (c). The equivalent for $\mathrm{E}_{\mathrm{y}}$ are shown in (b) and (d) for $3 \mathrm{~km}$ and $6 \mathrm{~km}$ offline data.

Figure 4: Sensitivity to the anisotropy is shown for $E_{x}, E_{y}$ components and for $P_{\max }$ for models 1, 2 and 3. The white contour marks where these sensitivities are equal to 1 and the grey contours mark the $10^{-15} \mathrm{~V} / \mathrm{Am}^{2}$ (inner) and the $10^{-16} \mathrm{~V} / \mathrm{Am}^{2}$ (outer) noise floor. Red arrows show the HED position.

Figure 5: The peak sensitivity extracted from the contour plots for model 2 is plotted against frequency. The values are plotted for a range of anisotropy ratios indicated by the variation of $\mathrm{R}_{\text {horz }}$ from 1 to $20 \Omega \mathrm{m}$ while $\mathrm{R}_{\mathrm{vert}}$ is fixed at $40 \Omega \mathrm{m}$. Figure (a) shows the $\mathrm{E}_{\mathrm{x}}$ component data and (b) $\mathrm{E}_{\mathrm{y}}$.

Figure 6: Sensitivity to the anisotropy for $E_{x}, E_{y}$ components and for $P_{\max }$ for the 3D slab model. The white contour marks where these sensitivities are equal to 1 and the grey contours mark the $10^{-15} \mathrm{~V} / \mathrm{Am}^{2}$ and the $10^{-16} \mathrm{~V} / \mathrm{Am}^{2}$ noise floors of the data. Red arrows show the source dipole position.

Figure 7: Probability density functions obtained from stochastic inversion for the isotropic target layer, $\left(100 \mathrm{~m}\right.$ thick, $\mathrm{R}_{\text {horz }}=\mathrm{R}_{\text {vert }}=40 \Omega \mathrm{m}$ ). (a) Inline (black) and offline (red) pdfs for $\mathrm{R}_{\text {horz. }}$ (b) Inline (black) and offline (red) pdfs for $\mathrm{R}_{\mathrm{vert}}$. True values shown as a blue line.

Figure 8: Probability density functions obtained from stochastic inversion for the anisotropic target layer, (100 m thick layer, $\mathrm{R}_{\text {horz }}=1, \mathrm{R}_{\mathrm{vert}}=40 \Omega \mathrm{m}$ ). (a) Inline (black) and offline (red) pdfs for $\mathrm{R}_{\text {horz. }}$ (b) Inline (black) and offline (red) pdfs for $\mathrm{R}_{\mathrm{vert}}$. True values shown as a blue line.

Figure 9: Percentage difference $\mathrm{SPD}_{\mathrm{PD}}$, the sensitivity to variation in $\mathrm{R}_{\text {horz }}$ normalized by the 


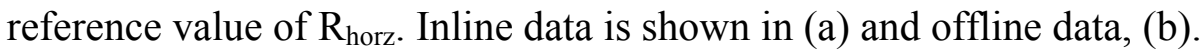

Figure 10: Depth section ( $x-z$ plane) through the reservoir. Polarization ellipses are shown for the isotropic reservoir case in black and the anisotropic reservoir in white. Shaded colors indicate the strength of the polarization-ellipse maximum (anisotropic case). (a) The electric field polarization-ellipses. (b) to (d) The current density polarization ellipses for increasing anisotropies (colorbar for (b) is the same as (c)-(d)).

Figure 11: Horizontal and vertical current density in the reservoir at $\mathrm{x}=3 \mathrm{~km}$ is plotted against horizontal resistivity.

Figure 12: $x-y$ cross section at 2:1 vertical exaggeration through $3 \mathrm{D}$ anisotropic inversion of the isotropic target model. Maximum offline separation is $8 \mathrm{~km}$ with $10^{-16} \mathrm{~V} / \mathrm{Am}^{2}$ noise floor. Top panel shows vertical resistivity, bottom panel shows horizontal resistivity (x-direction). Location of $3 \mathrm{D}$ anisotropic target is shown by black outline and receiver positions are shown as black triangles.

Figure 13: Depth slices through the 3D anisotropic inversion of the isotropic target model. Maximum offline separation is $8 \mathrm{~km}$ with $10^{-16} \mathrm{~V} / \mathrm{Am}^{2}$ noise floor. Top panel shows vertical resistivity for depth slice $4600-4700 \mathrm{~m}$. Bottom panel shows horizontal resistivity in the $\mathrm{x}-$ direction for depth slice 3900-4000m (location of largest differences from background).

Figure 14: $x-y$ cross section through 3D anisotropic inversion of the anisotropic target model. Maximum offline separation is $8 \mathrm{~km}$ with $10^{-16} \mathrm{~V} / \mathrm{Am}^{2}$ noise floor. Top panel shows vertical resistivity, bottom panel shows horizontal resistivity (x-direction). Location of the $3 \mathrm{D}$ anisotropic target is shown by black outline and receiver positions are shown as black triangles.

Figure 15: Top panel shows vertical resistivity for depth slice 4600-4600m. Maximum offline separation is $8 \mathrm{~km}$ with $10^{-16} \mathrm{~V} / \mathrm{Am}^{2}$ noise floor. Bottom panel shows horizontal resistivity in the $\mathrm{x}$-direction for depth slice 4300-4400m. Anisotropic target is outlined in black, with receiver array shown as black dots.

Figure 16: $x-y$ cross section at 2:1 vertical exaggeration through $3 \mathrm{D}$ anisotropic inversion of the isotropic target model. Maximum offline separation is $2 \mathrm{~km}$ with $10^{-15} \mathrm{~V} / \mathrm{Am}^{2}$ noise floor. Top panel shows vertical resistivity, bottom panel shows horizontal resistivity (X direction). Location of 3D anisotropic target shown is by black outline and receiver positions are shown as black triangles. 
Figure 1

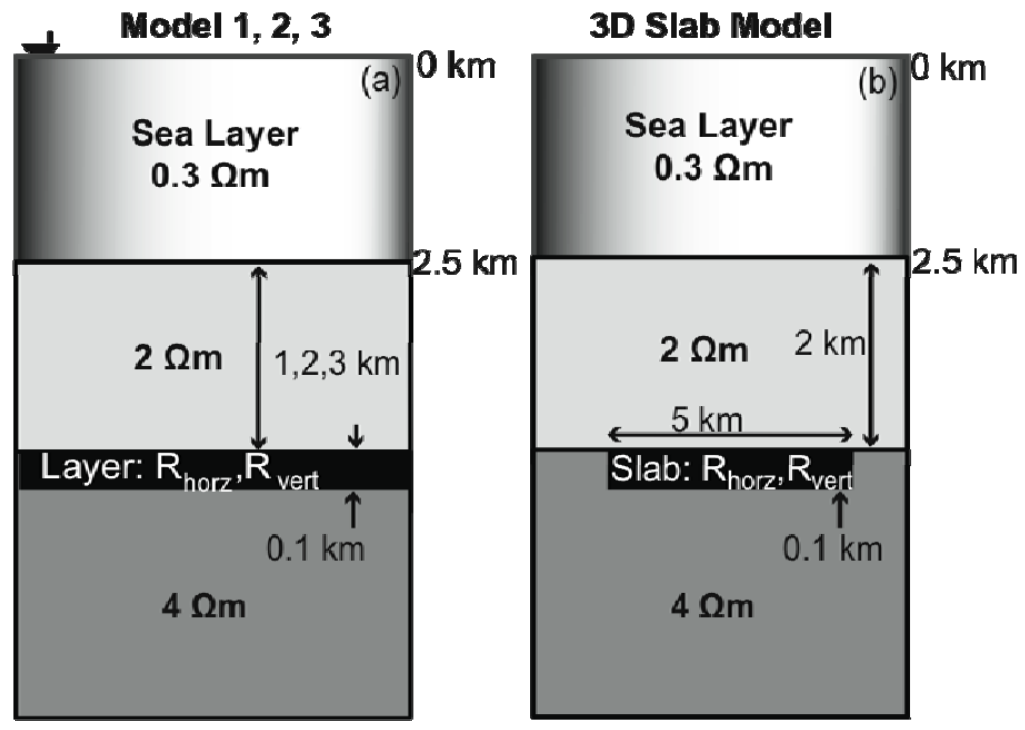


Figure 2
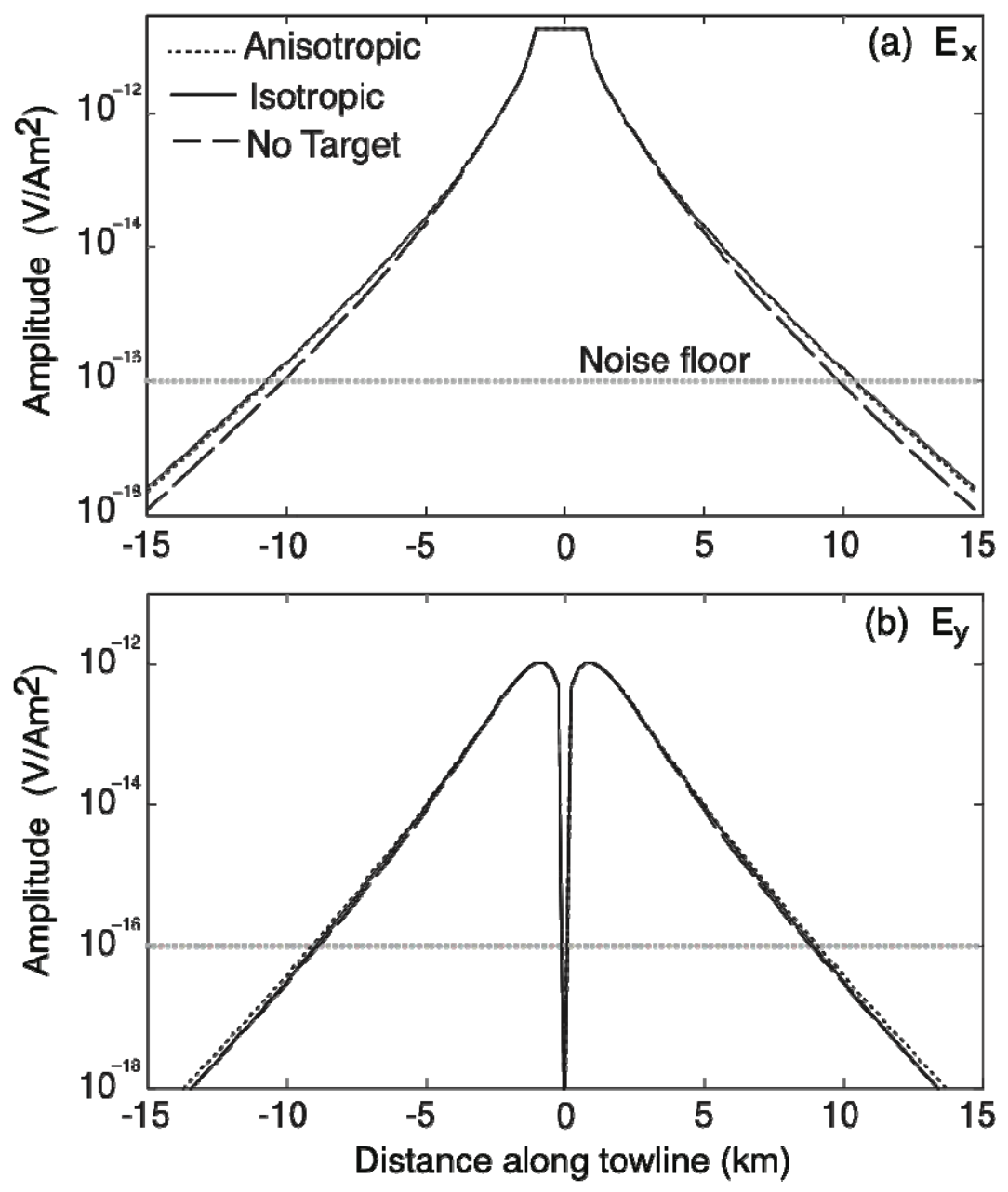
Figure 3
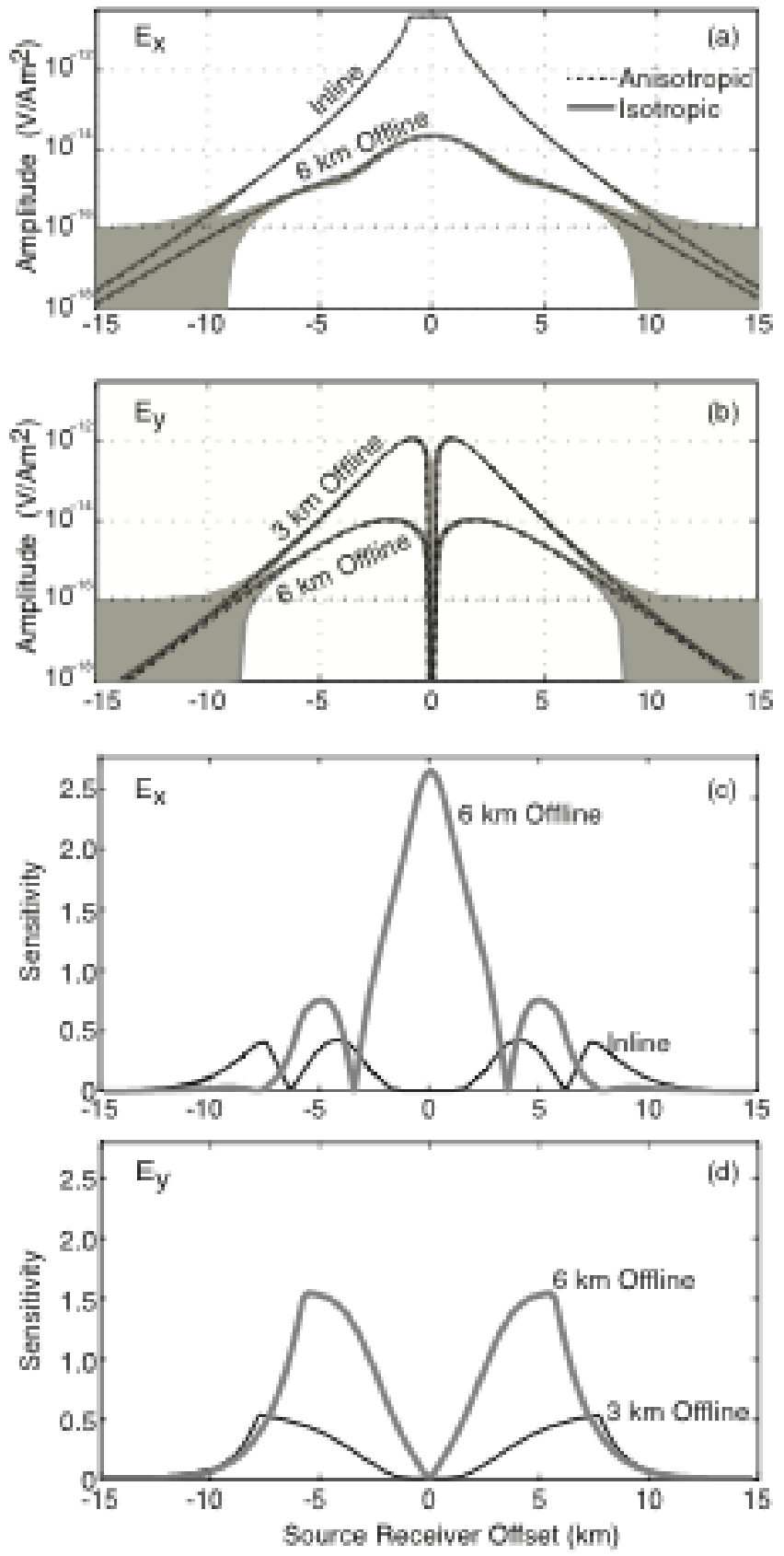
Figure 4
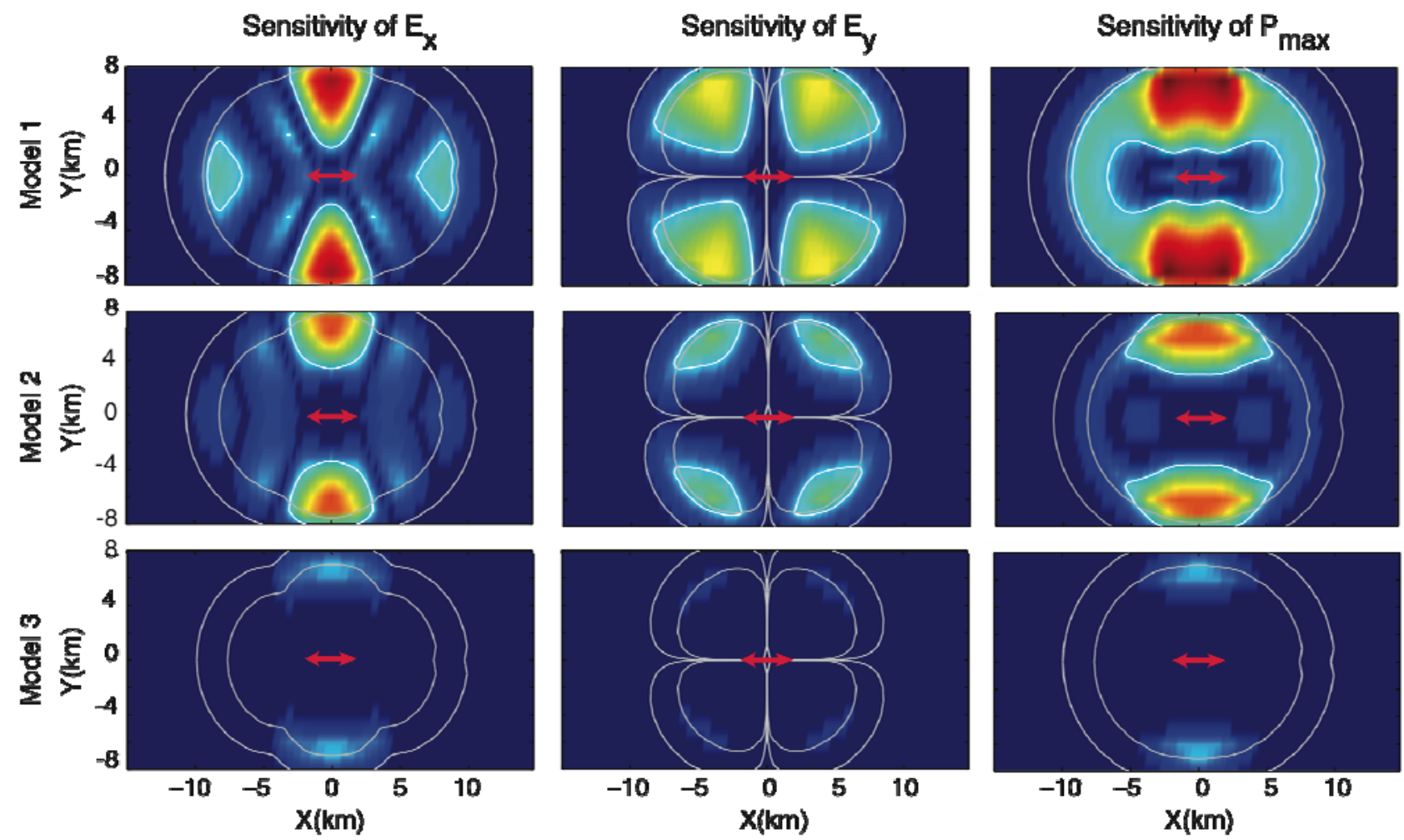

Sensitivity

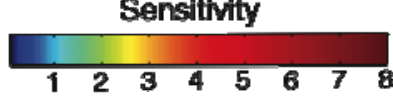


Figure 5
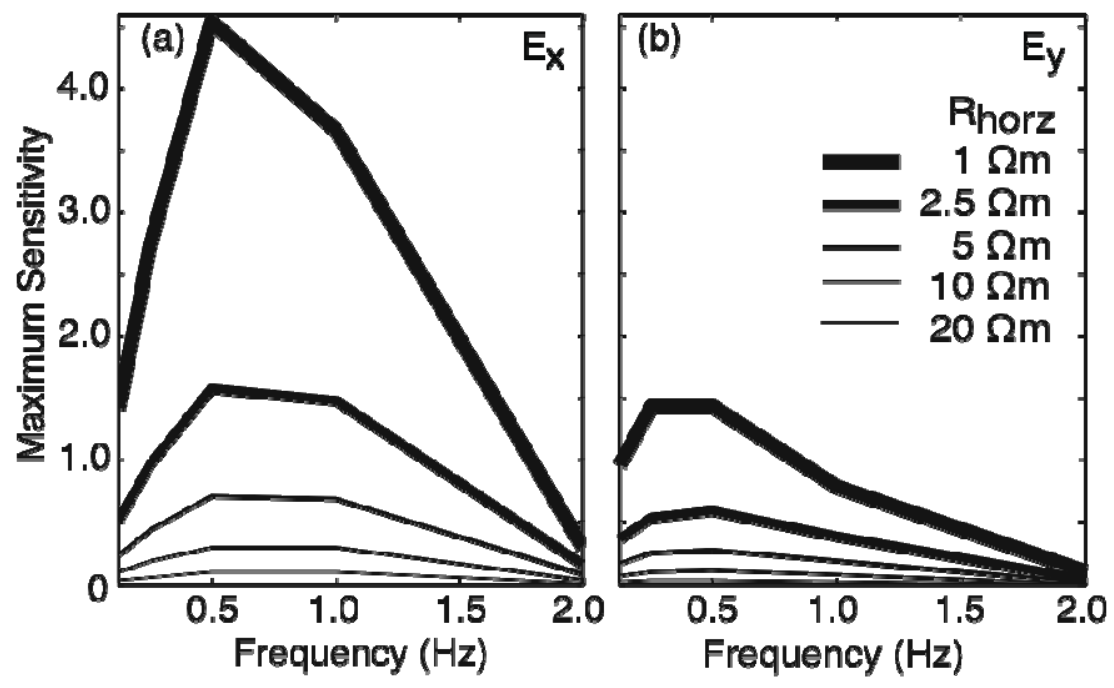
Figure 6
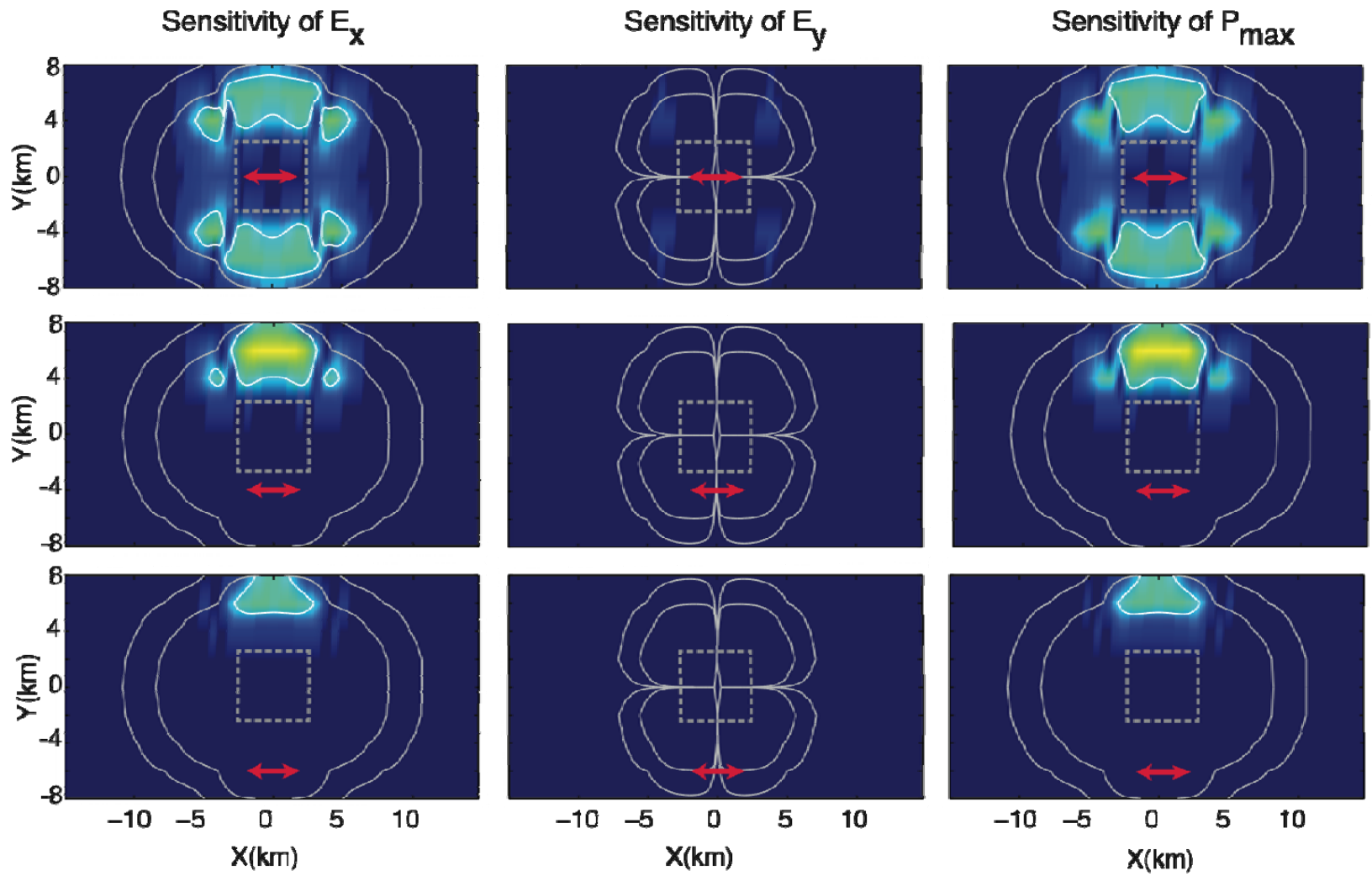

Sensitivity

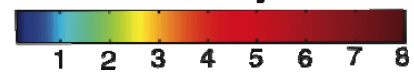


Figure 7

(a) Isotropic $R_{\text {horz }}$

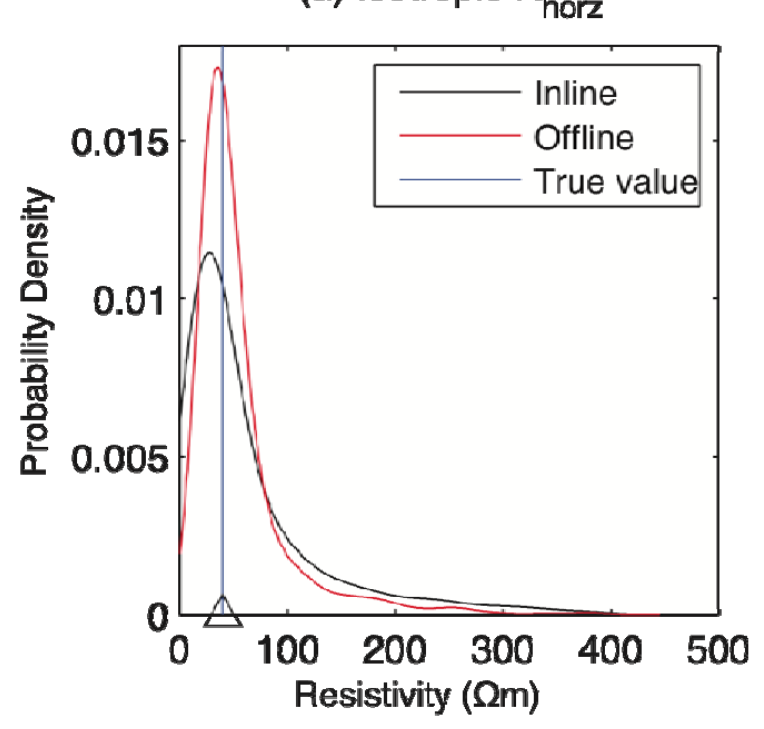

(b) Isatropic $\mathrm{R}_{\text {vert }}$

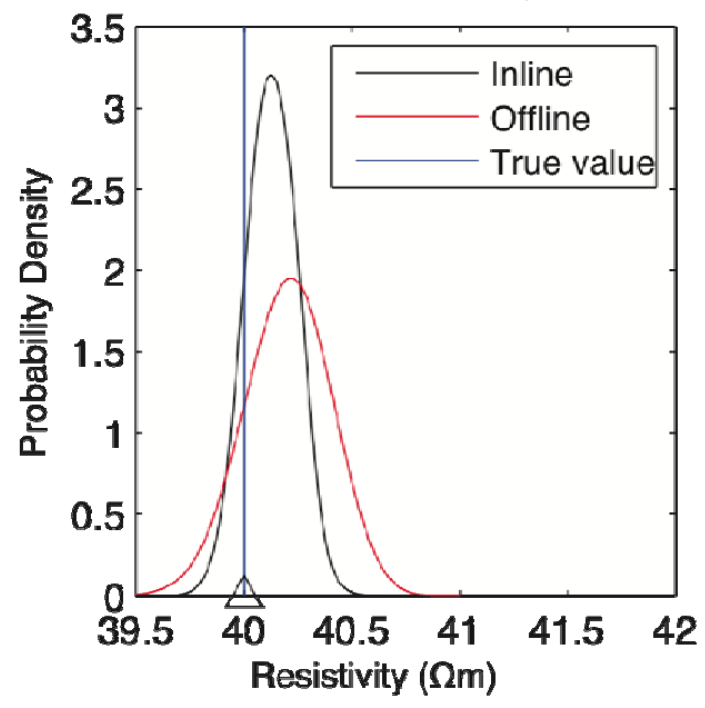


Figure 8

(a) Anisotropic $R_{\text {horz }}$

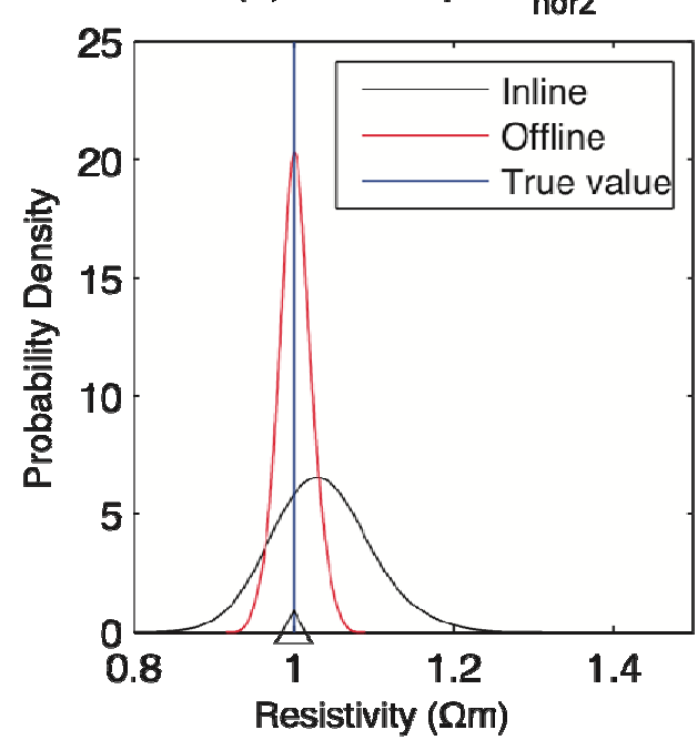

(b) Anisotropic $\mathrm{R}_{\text {vert }}$

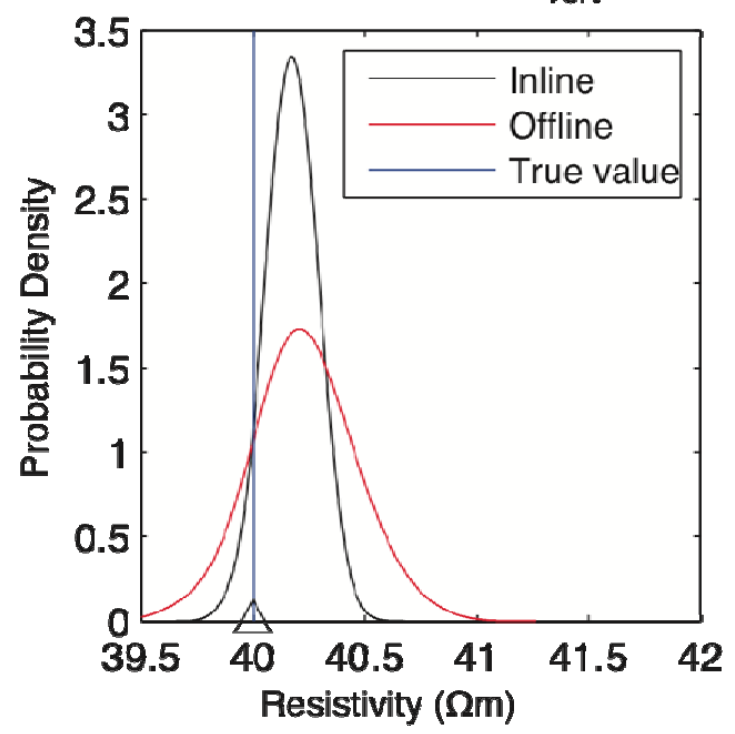


Figure 9

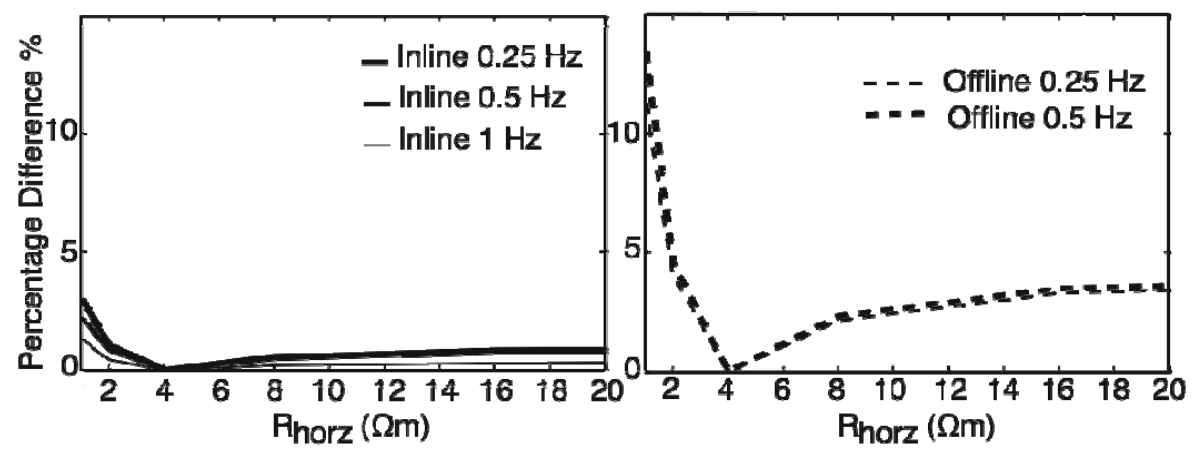




\section{Figure 10}

White : Anisotropic Target, $R_{\text {vert }}=40 \Omega m$, vary $R_{\text {horz }}$ (see each plot)

Black : Isotropic Target, $\mathrm{R}_{\text {horz }}=\mathrm{R}_{\text {vert }}=40 \Omega \mathrm{m}$
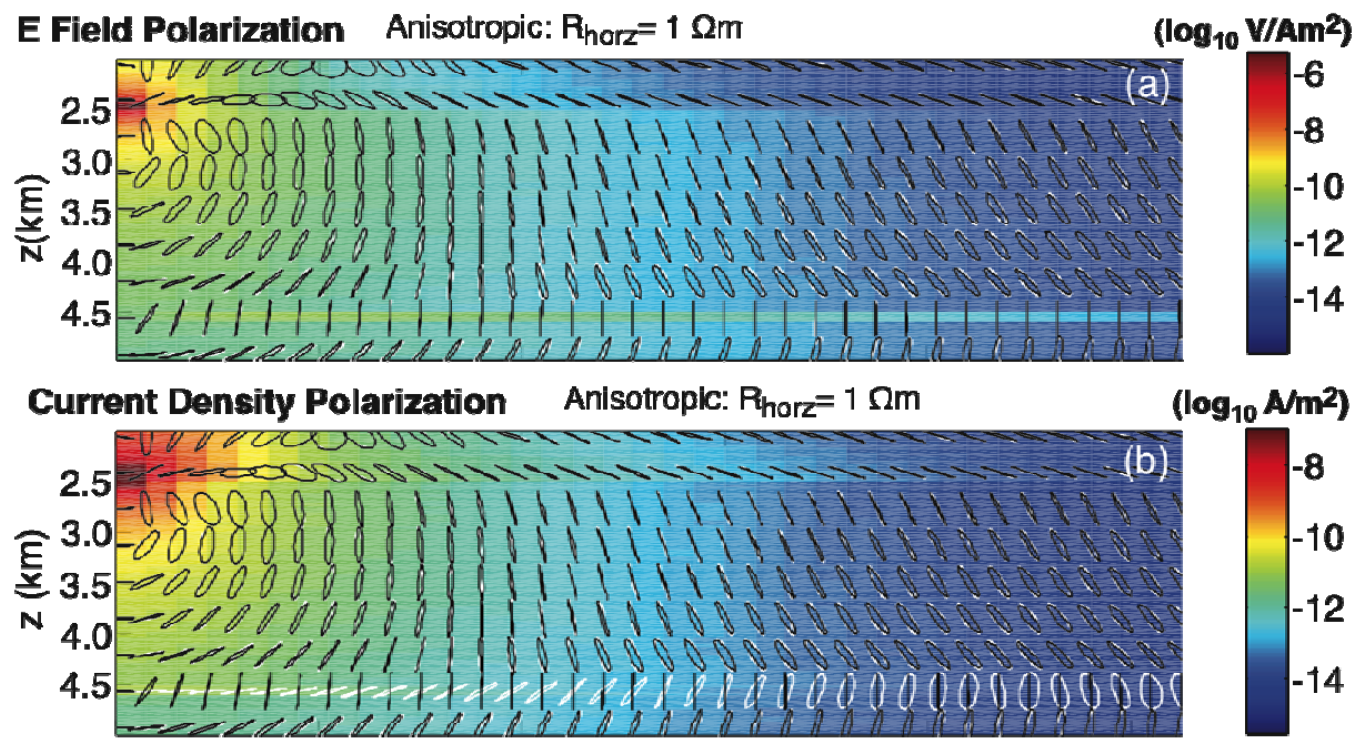

\section{Current Density Polarization Anisotropic: $\mathrm{P}_{\mathrm{horz}}=4 \Omega \mathrm{m}$}

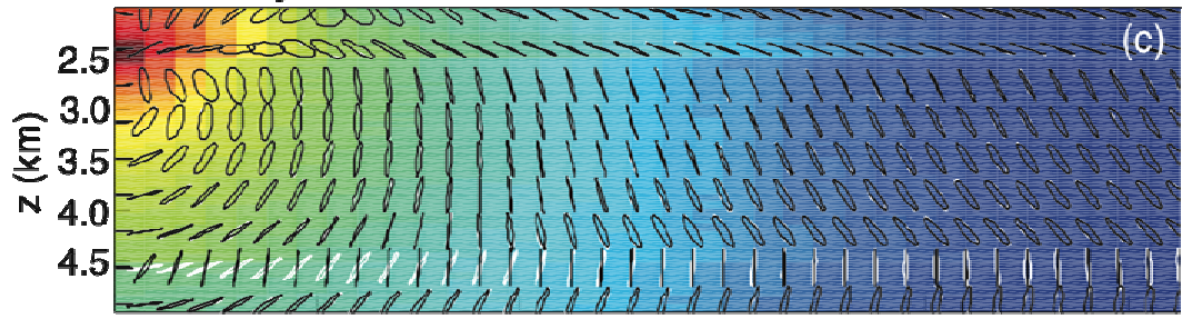

Current Density Polarization Anisotropic: $R_{\text {horz }}=16 \Omega \mathrm{m}$

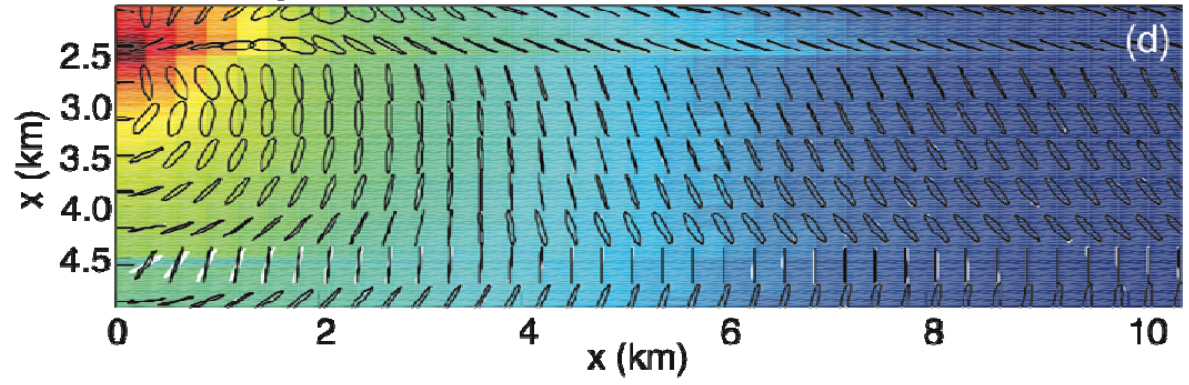


Figure 11

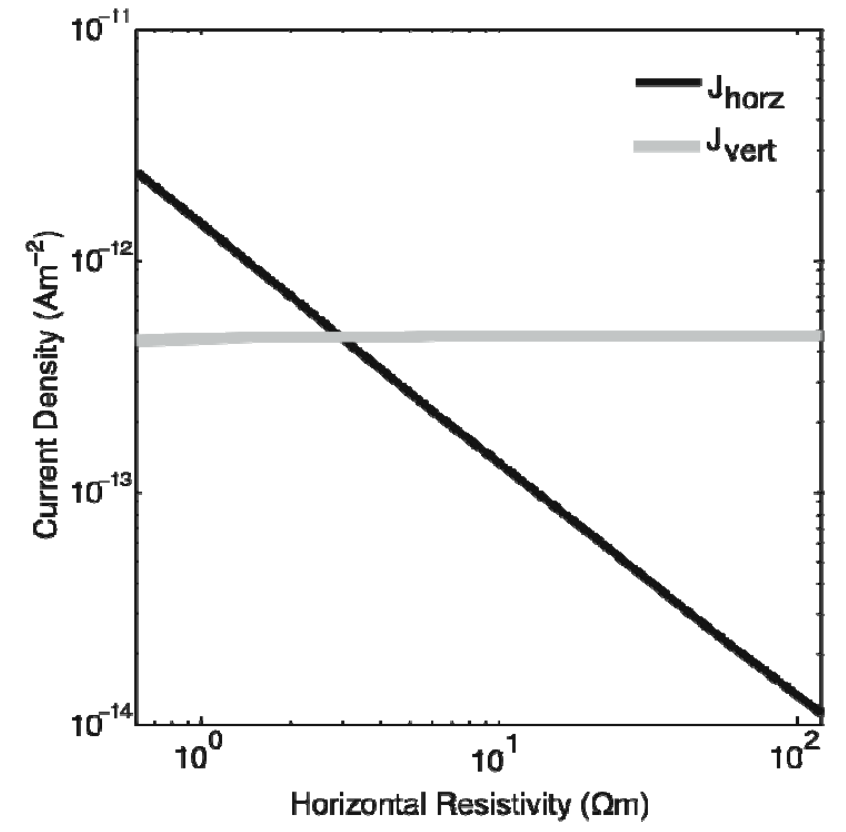


Figure 12
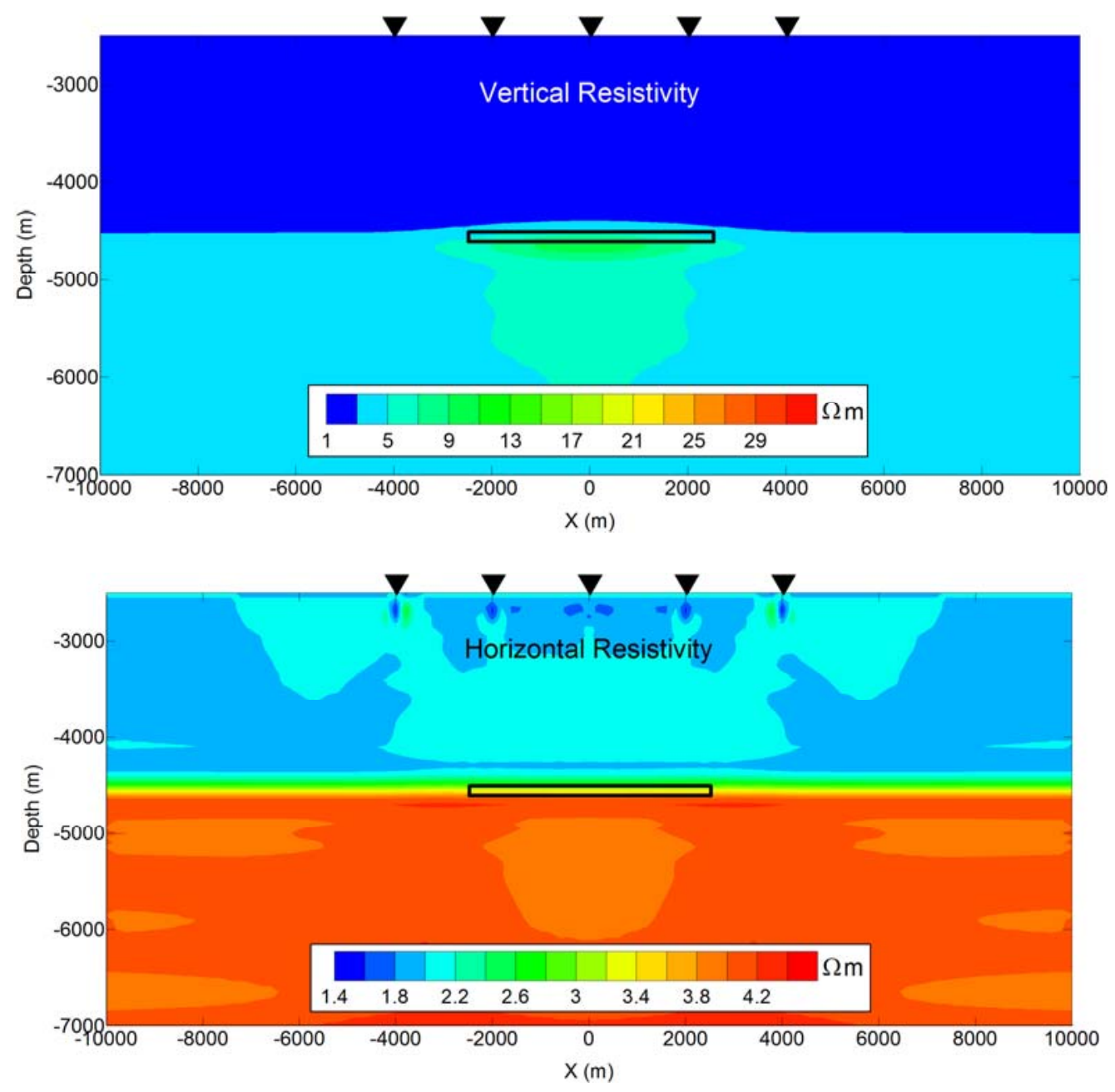


\section{Figure 13}
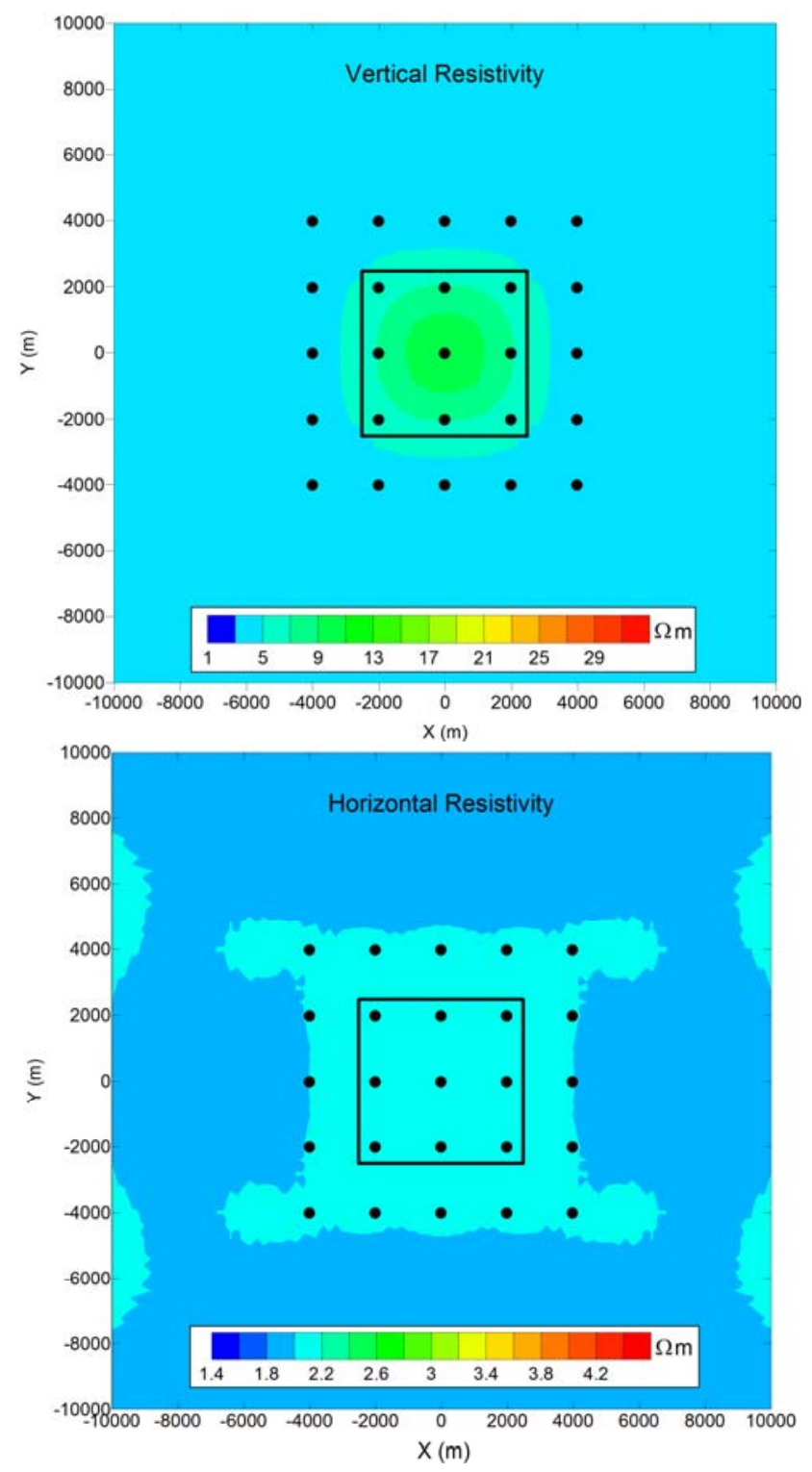
Figure 14

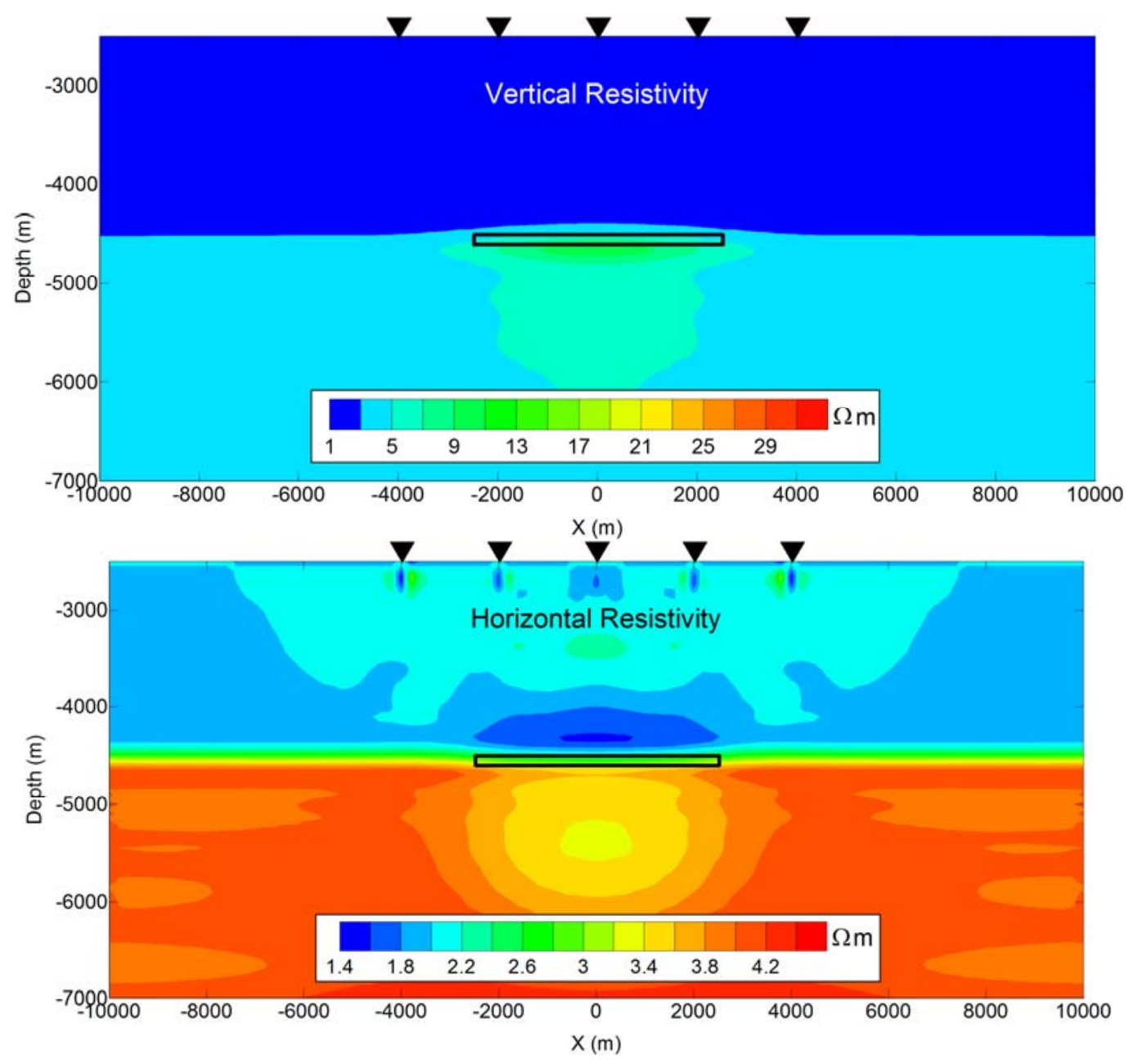


Figure 15
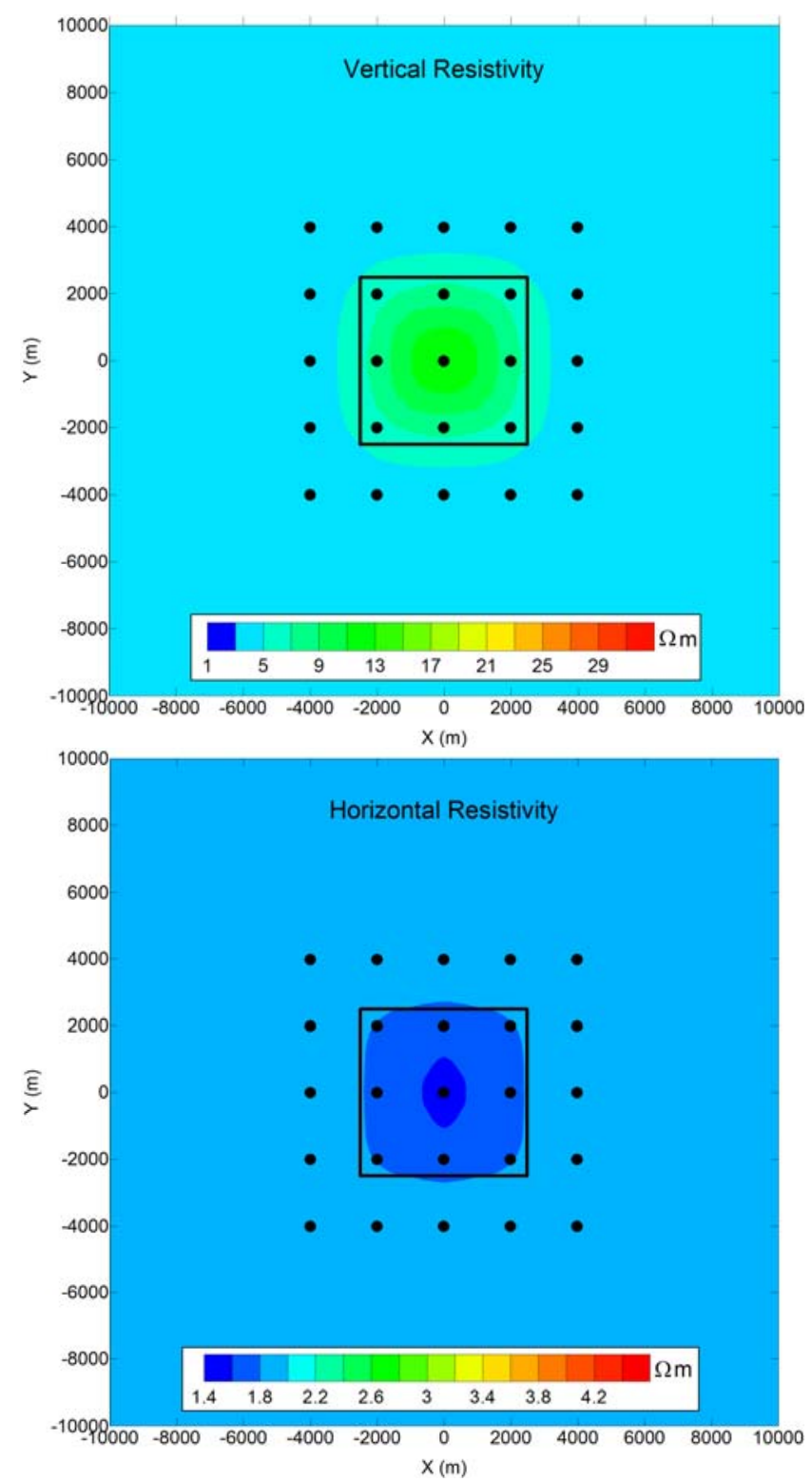


\section{Figure 16}

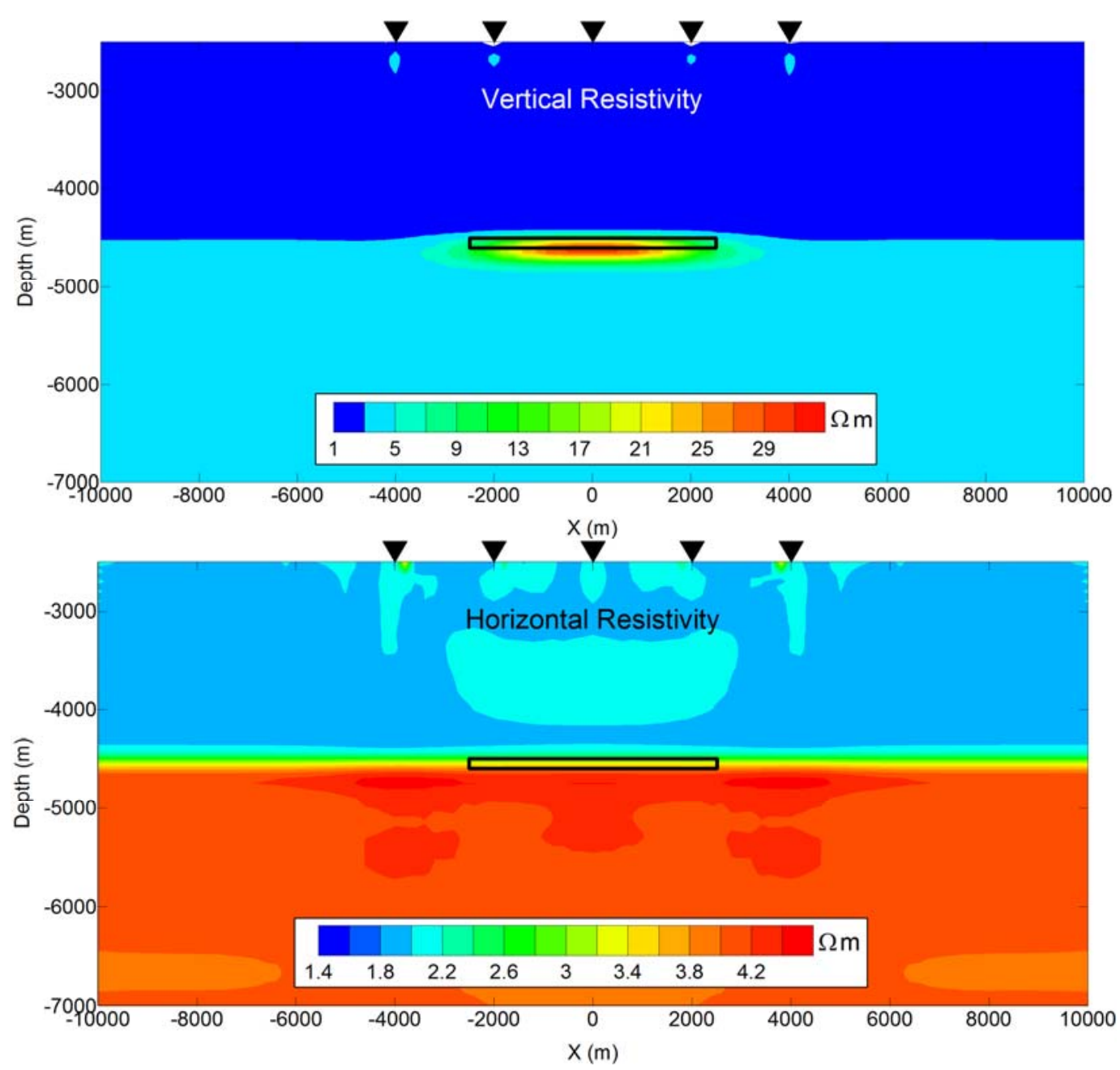




\section{DISCLAIMER}

This document was prepared as an account of work sponsored by the United States Government. While this document is believed to contain correct information, neither the United States Government nor any agency thereof, nor The Regents of the University of California, nor any of their employees, makes any warranty, express or implied, or assumes any legal responsibility for the accuracy, completeness, or usefulness of any information, apparatus, product, or process disclosed, or represents that its use would not infringe privately owned rights. Reference herein to any specific commercial product, process, or service by its trade name, trademark, manufacturer, or otherwise, does not necessarily constitute or imply its endorsement, recommendation, or favoring by the United States Government or any agency thereof, or The Regents of the University of California. The views and opinions of authors expressed herein do not necessarily state or reflect those of the United States Government or any agency thereof or The Regents of the University of California.

Ernest Orlando Lawrence Berkeley National Laboratory is an equal opportunity employer. 